\title{
Asymptotics for Bailey-type mock theta functions
}

\section{Taylor Garnowski ${ }^{1}$}

Received: 19 January 2021 / Accepted: 25 August 2021 / Published online: 26 December 2021

(c) The Author(s) 2021

\section{Abstract}

We compute asymptotic estimates for the Fourier coefficients of two mock theta functions, which come from Bailey pairs derived by Lovejoy and Osburn. To do so, we employ the circle method due to Wright and a modified Tauberian theorem. We encounter cancelation in our estimates for one of the mock theta functions due to the auxiliary function $\theta_{n, p}$ arising from the splitting of Hickerson and Mortenson. We deal with this by using higher-order asymptotic expansions for the Jacobi theta functions.

Keywords Mock theta functions · Bailey pairs · Wright circle method

Mathematics Subject Classification 05A17 $\cdot 11 \mathrm{M} 45 \cdot 11 \mathrm{P} 55 \cdot 11 \mathrm{P} 82$

\section{Introduction}

\subsection{History}

The classical study of mock theta functions originated with Ramanujan in the early 20 th century and continues to the present day. There are varying versions of definitions for what a classical mock theta function is (see for example, $[7,13]$ ), but they generally all encompass the following: Let $q$ be a complex variable with $|q|<1$. A classical mock theta function $M(q)$ is a function for which near each root of unity $\xi$ there exists a weakly holomorphic modular form, $F_{\xi}$, and a rational number $a_{\xi}$, such that near $\xi$

$$
M(q)-q^{\alpha_{\xi}} F_{\xi}(q)=O(1) .
$$

We then eliminate the possibility of having holomorphic theta functions from the definition by declaring that no $F_{\xi}$ satisfies the above condition for all roots of unity. A nice list of the classical mock theta functions exists in the appendix of [7] and Sect.

\footnotetext{
$凶$ Taylor Garnowski

tgarnows@uni-koeln.de

1 Universität zu Köln, Cologne, Germany
} 
4 of [15]. Large families of new examples of modular type functions that satisfy Eq. (1.1) were discovered after Zwegers wrote his thesis [21] on mock theta functions in 2002, whereby the classical mock theta functions were found to be linked to harmonic Maass forms. From [21], we can define a mock theta function to be the holomorphic part of a weight $\frac{1}{2}$ harmonic Maass form (see [7] for more on harmonic Maass forms), and thus, functions that are finite sums of normalized Appell sums can be viewed as mock theta functions. This result brings the theory of mock theta functions and combinatorial generating functions closer together. For example, let $\zeta:=e^{2 \pi i z}$, then the famous partition rank generating function,

$$
R(z ; \tau):=\sum_{n \geq 0} \frac{q^{n^{2}}}{\left(\zeta q, \zeta^{-1} q ; q\right)_{n}}:=\sum_{n=0}^{\infty} \sum_{m \in \mathbb{Z}} N(m, n) \zeta^{m} q^{n}
$$

can be written as a sum of normalized Appell sums and is, thus, a mock theta function when $z \in \mathbb{H}$ (Lemma 3.1 in [10]). Understanding how the coefficients of mock theta functions grow is important, especially when a combinatorial interpretation is available. For example (see Theorem 1.2 in [10]),

$$
N(m, n) \sim \frac{\beta}{4} \operatorname{sech}\left(\frac{\beta m}{2}\right) p(n)=\frac{\beta}{16 \sqrt{3} n} \operatorname{sech}\left(\frac{\beta m}{2}\right) e^{\pi \sqrt{\frac{2 n}{3}}},
$$

where $p(n)$ is the partition function, $\beta:=\frac{\sqrt{n} \log (n)}{\pi \sqrt{6}}$, and $f(n) \sim g(n)$ denotes that the ratio of $f(n)$ and $g(n)$ goes to 1 as $n \rightarrow \infty$.

\subsection{Bailey pairs and mock theta functions}

The inspiration for this work comes from the fact that we want to find similar asymptotic estimates for mock theta functions that come from Bailey pairs. Let $\alpha_{n}(q)=: \alpha_{n}$ and $\beta_{n}(q)=: \beta_{n}$ be two sequences of $q$-series. The tuple $\left(\alpha_{n}, \beta_{n}\right)$ is referred to as a Bailey pair with respect to $a \in \mathbb{C}$ (assuming $a$ causes no poles in what follows) if

$$
\beta_{n}=\sum_{k=0}^{n} \frac{\alpha_{k}}{(q)_{n-k}(a q)_{n+k}}
$$

where $(a ; q)_{n}:=\prod_{j=0}^{n-1}\left(1-a q^{j}\right),(a ; q)_{\infty}:=\prod_{j=0}^{\infty}\left(1-a q^{j}\right)$, and $(q ; q)_{n}=:(q)_{n}$ are the usual $q$-Pochammer symbols. The fact that Bailey pairs and mock theta functions are related is not immediately obvious, and it was not until Andrews showed that Eq. (1.2) can be iterated to obtain an infinite family of Bailey pairs that a true connection was found [1,2]. This is the content of Bailey's lemma [1-4]. Bailey's lemma leads to families of sums, known as higher-level Appell sums, which are not necessarily mock theta functions, but a more general object called a mixed mock theta function $[7,19]$. Occasionally, certain pairs lead to normal Appell sums via Bailey's lemma, and we call the resulting functions Bailey-type mock theta functions. 
The study of Bailey-type mock theta functions became more interesting with a key result by Hickerson and Mortenson [15], which gave an explicit decomposition of indefinite theta functions in terms of Appell sums and theta functions. This result was used by many authors in works such as $[14,18,19]$ to write families of Bailey-type mock theta functions in terms of classical mock theta functions. For example, Lovejoy and Osburn in [19] derive a Bailey-type mock theta function, $R_{1}^{(4)}(q)$, and used the decomposition of [15] to find the formula:

$$
R_{1}^{(4)}(q)=-\phi\left(q^{4}\right)+M_{1}(q)
$$

where $\phi$ is the 10th-order classical mock theta function given by

$$
\phi(q):=\sum_{n=0}^{\infty} \frac{q^{\frac{n(n+1)}{2}}}{\left(q ; q^{2}\right)_{n+1}}
$$

and $M_{1}(q)$ is a weakly holomorphic modular form. Understanding how the coefficients of certain Bailey-type mock theta functions grow is an interesting question, which was proposed by Lovejoy and Osburn in [19], and which we will begin to answer in this work. To the best of our knowledge, no works have investigated the growth of Baileytype mock theta functions in depth. Doing so here for two example functions, we hope to lay the groundwork for future and more advanced studies of the asymptotic properties of Bailey-type mock theta functions. Let $a(n)$ denote the coefficients of $R_{3}^{(3)}$ and $b(n)$ the coefficients of $R_{1}^{(3)}$, which are two Bailey-type mock theta functions defined in Definition 2.2 (the $a(n)$ and $b(n)$ are explicitly defined in Examples 1 and 2). We will show the following.

Theorem 1 The following estimates hold as $n \rightarrow \infty$ :

$$
\begin{aligned}
& a(n) \sim(-1)^{n} \frac{\sqrt{6}}{12 \sqrt{n}} e^{\pi \sqrt{\frac{n}{12}}}, \\
& b(n) \sim\left(\frac{1}{2 \sin \left(\frac{\pi}{4}\right) \sin \left(\frac{5 \pi}{12}\right)}+1\right) \frac{e^{\pi \sqrt{\frac{n}{6}}}}{\sqrt{24 n}} .
\end{aligned}
$$

The following table shows the ratio between the estimated values in Theorem 1 and the actual values for some values of $n$.

\begin{tabular}{lll}
\hline$n$ & $a(n)$ & $b(n)$ \\
\hline 100 & 0.96315 & 0.98067 \\
500 & 0.98249 & 0.99081 \\
1000 & 0.98740 & 0.99343 \\
\hline
\end{tabular}


To obtain asymptotic estimates, like the ones we give in our main Theorem 1, it is often useful to use a modified circle method due to Wright [20], which allows one to look at a finite number of poles. Wright's technique has been used by several authors in recent years $[5,6,10,12]$ to deal with combinatorial generating functions like $R(z ; \tau)$, for example. The common theme here and in the works $[5,6,10,12]$ is that the functions are generically mixed mock modular forms, which are more suited for the adapted circle method of Wright.

This work is organized as follows: In Sect. 2, we define the main objects of this work. In Sect. 3, we provide estimates near $\tau=0$ of the Jacobi theta function and normalized Appell sum. In Sect. 4, we employ the Wright circle method to prove the first part of our main theorem, and in Sect. 5, we use results from $[8,16]$ to prove the second part of our theorem. Finally, we offer some remarks on our results and thoughts on future work regarding this topic in Sect. 6.

\section{Preliminaries and basic definitions}

The basic objects that appear in this work, and some of their properties, are collected in this section. We begin by recalling that the definitions of the normalized Appell sum and the Jacobi theta function:

$$
\mu\left(z_{1}, z_{2} ; \tau\right):=\frac{\zeta_{1}^{\frac{1}{2}}}{\vartheta\left(z_{2} ; \tau\right)} \sum_{n \in \mathbb{Z}} \frac{(-1)^{n} q^{\frac{n^{2}+n}{2}} \zeta_{2}^{n}}{1-q^{n} \zeta_{1}}
$$

where $z_{1}, z_{2} \in \mathbb{C}, \zeta_{j}:=e^{2 \pi i z_{j}}, q:=e^{2 \pi i \tau}, \tau \in \mathbb{H}$, and $\vartheta$ is the Jacobi theta function (or $\vartheta$-function, for short) given by

$$
\vartheta\left(z_{2} ; \tau\right):=\sum_{m \in \frac{1}{2}+\mathbb{Z}}(-1)^{m} q^{\frac{m^{2}}{2}} \zeta_{2}^{m} .
$$

Furthermore, we have the Jacobi product representation for the $\vartheta$-function:

$$
\vartheta(z ; \tau)=-i q^{\frac{1}{8}} \zeta^{-\frac{1}{2}}(\zeta ; q)_{\infty}(q ; q)_{\infty}\left(q \zeta^{-1} ; q\right)_{\infty}
$$

where $\zeta:=e^{2 \pi i z}$. Many of the important functions discussed here were originally defined in [19] and [15]. In those works, the authors use a slightly different notation for the $\vartheta$-function (denoted by $j$ ) and the Appell sum (denoted by $m$ ). One can go between the two via the formulas:

$$
\begin{aligned}
\vartheta\left(z_{2} ; \tau\right) & =-i q^{1 / 8} \zeta_{2}^{-1 / 2} j\left(\zeta_{2}, q\right), \\
m\left(\zeta_{1} ; q ; \zeta_{2}\right) & =i q^{1 / 8} \zeta_{1}^{-1 / 2} \mu\left(z_{1}+z_{2}, z_{2} ; \tau\right) .
\end{aligned}
$$

We will use the following identities frequently in this work. 
Proposition 2.1 (see Chap. 1 of [21]) The normalized Appell sum and Jacobi $\vartheta$ function satisfy:

1. $\mu\left(z_{1}, z_{2} ; \tau+1\right)=e^{-\frac{\pi i}{4}} \mu\left(z_{1}, z_{2} ; \tau\right)$,

2. $\mu\left(z_{1}+1, z_{2} ; \tau\right)=\mu\left(z_{1}, z_{2}+1 ; \tau\right)=-\mu\left(z_{1}, z_{2} ; \tau\right)$,

3. $\mu\left(\frac{z_{1}}{\tau}, \frac{z_{2}}{\tau} ;-\frac{1}{\tau}\right)=-\sqrt{-i \tau} e^{-\pi i \frac{\left(z_{1}-z_{2}\right)^{2}}{\tau}} \mu\left(z_{1}, z_{2} ; \tau\right)+\frac{\sqrt{-i \tau}}{2 i} e^{-\pi i \frac{\left(z_{1}-z_{2}\right)^{2}}{\tau}} h\left(z_{1}-\right.$ $\left.z_{2} ; \tau\right)$, where $h(z ; \tau)$ is the Mordell integral given by

$$
h(z ; \tau):=\int_{\mathbb{R}} \frac{e^{\pi i \tau x^{2}} e^{-2 \pi z x}}{\cosh (\pi x)} d x,
$$

4. $h\left(\frac{z}{\tau} ;-\frac{1}{\tau}\right)=\sqrt{-i \tau} e^{-\frac{\pi i z^{2}}{\tau}} h(z ; \tau)$,

5. $\vartheta(z+\tau ; \tau)=-e^{-\pi i \tau-2 \pi i z} \vartheta(z, \tau)$,

6. $\vartheta(z ; \tau+1)=e^{\frac{\pi i}{4}} \vartheta(z ; \tau)$,

7. $\vartheta\left(\frac{z}{\tau} ;-\frac{1}{\tau}\right)=-i \sqrt{-i \tau} e^{\frac{\pi i z^{2}}{\tau}} \vartheta(z ; \tau)$, and

8. $\eta(\tau)=\frac{1}{\sqrt{-i \tau}} \eta\left(-\frac{1}{\tau}\right)$ and $\eta(\tau+1)=e^{\frac{\pi i}{12}} \eta(\tau)$, where $\eta$ is Dedekind's eta function.

For $k \geq 3$ Lovejoy and Osburn showed that the following family of functions are mock theta functions.

Definition 2.2 ([19]) Let $k \geq 3$ and $n_{1}, \ldots, n_{k}$ be integers such that $1 \leq n_{1} \ldots \leq n_{k}$. Define

$$
\begin{aligned}
B_{k}\left(n_{k}, n_{k-1}, \ldots, n_{1} ; q\right):= & (-1)^{n_{1}}(-q)_{n_{k-1}} q^{\left(\begin{array}{c}
n_{k-1}+1 \\
2
\end{array}\right)} \\
& \times \frac{\prod_{j=2}^{k-1} q^{2^{j-2} n_{k-j}}\left(-q^{2^{j-2}} ; q^{2^{j-2}}\right)_{2 n_{k-j}}}{\prod_{j=1}^{k}\left(q^{2^{j-1}} ; q^{2^{j-1}}\right)_{n_{k-j+1}-n_{k-j}}},
\end{aligned}
$$

with $n_{0}:=0$. Then we define

$$
\begin{aligned}
& R_{1}^{(k)}(q):=\sum_{n_{k} \geq n_{k-1} \geq \ldots \geq n_{1} \geq 0} q^{\left(\begin{array}{c}
n_{k}+1 \\
2
\end{array}\right)} B_{k}\left(n_{k}, \ldots, n_{1} ; q\right), \\
& R_{3}^{(k)}(q):=\sum_{n_{k} \geq n_{k-1} \geq \ldots \geq n_{1} \geq 0} \frac{(-1)^{n_{k}} q^{n_{k}^{2}+2 n_{k}}\left(q ; q^{2}\right) n_{k}}{\left(-q^{2} ; q^{2}\right)_{n_{k}}} B_{k}\left(n_{k}, \ldots, n_{1} ; q^{2}\right) .
\end{aligned}
$$

The authors of [19] showed that

$$
R_{1}^{(3)}(q)=v(-q)
$$

where $v(q):=\sum_{n \geq 0} \frac{q^{n^{2}+n}}{\left(-q ; q^{2}\right)_{n+1}}$ is a classical third order mock theta function. 
The first definition comes from the work of [15] and uses the standard combinatorial notation for the Jacobi triple product

$$
j(x, q):=(x)_{\infty}\left(q x^{-1}\right)_{\infty}(q)_{\infty},
$$

where $x$ is a non-zero complex number. When $x$ an integral or half integral power of $q$, we will always write $j$ in terms of a $\vartheta$-function, as discussed in Sect. 2, via the transformations

$$
\begin{aligned}
\vartheta(a \tau ; b \tau) & =-i q^{\frac{b}{8}} q^{-\frac{a}{2}} j\left(q^{a}, q^{b}\right), \\
\vartheta\left(a \tau+\frac{1}{2} ; b \tau\right) & =-q^{\frac{b}{8}} q^{-\frac{a}{2}} j\left(-q^{a}, q^{b}\right) .
\end{aligned}
$$

Definition 2.3 (see Sect. 2, [19] and Theorem 1.3, [15]) Let $x$ and $y$ be complex numbers so that they do not cause poles in the quotients that follow. Then for positive integers $n, p, r:=r^{*}+\left\{\frac{(n-1)}{2}\right\}$ and $s:=s^{*}+\left\{\frac{(n-1)}{2}\right\}$, with $\{a\}$ denoting the fractional part of the number $a$, define the function $\theta_{n, p}(x, y, q)$ by

$$
\begin{aligned}
\theta_{n, p}(x, y, q) \\
:=\frac{j^{3}\left(q^{p^{2}(2 n+p)}, q^{3 p^{2}(2 n+p)}\right)}{j\left(-1, q^{n p(2 n+p)}\right)} \\
\quad \times\left\{\sum_{r^{*}=0}^{p-1} \sum_{s^{*}=0}^{p-1} q^{n\left(\left(^{r-\frac{(n-1)}{2}}\right)+(n+p)\left(r-\frac{(n-1)}{2}\right)\left(s+\frac{(n+1)}{2}\right)+n\left(\left(^{s+\frac{(n+1)}{2}}\right)\right.\right.}\right. \\
\quad \times(-x)^{r-\frac{n-1}{2}}(-y)^{s+\frac{(n+1)}{2}} \\
\quad \times \frac{j\left(-q^{p n(s-r)} \frac{x^{n}}{y^{n}}, q^{n p^{2}}\right) j\left(q^{p(2 n+p)(r+s)+p(n+p)} x^{p} y^{p}, q^{p^{2}(2 n+p)}\right)}{j\left(q^{p r(2 n+p)+\frac{p(n+p)}{2}} \frac{(-y)^{n+p}}{(-x)^{n}}, q^{p^{2}(2 n+p)}\right) j\left(q^{p s(2 n+p)+\frac{p(n+p)}{2}} \frac{(-y)^{n+p}}{(-x)^{n}}, q^{\left.p^{2}(2 n+p)\right)}\right\},},
\end{aligned}
$$

where $\left(\begin{array}{l}b \\ c\end{array}\right)$ is the standard binomial coefficient.

Recall that

$$
m\left(\zeta_{1} ; q ; \zeta_{2}\right)=i q^{1 / 8} \zeta_{1}^{-1 / 2} \mu\left(z_{1}+z_{2}, z_{2} ; \tau\right)
$$

We then have the following theorem.

Theorem 2 ([19]) For $k \geq 3$ the function $R_{3}^{(k)}(q)$ is a mock theta function and satisfies the formula

$$
\begin{aligned}
R_{3}^{(k)}(q)= & 2 q^{-2^{k-3}\left(2^{k-2}+1\right)} m\left(q^{2^{k-2}}, q^{2^{2 k-2}+2^{k}},-1\right) \\
& -2 q^{-\frac{1}{8}} \frac{\theta_{1,4}\left(q^{2^{k-2}+1},-q^{2^{k-2}+1}, q\right)}{\vartheta\left(\frac{1}{2} ; \tau\right)}
\end{aligned}
$$




$$
\begin{gathered}
=2 i q^{-2^{k-3}} \mu\left(2^{k-2} \tau+\frac{1}{2}, \frac{1}{2} ;\left(2^{2 k-2}+2^{k}\right) \tau\right) \\
-2 q^{-\frac{1}{8}} \frac{\theta_{1,4}\left(q^{2^{k-2}+1},-q^{2^{k-2}+1}, q\right)}{\vartheta\left(\frac{1}{2} ; \tau\right)} .
\end{gathered}
$$

Example 2.4 (The function $R_{3}^{(3)}$ )

$$
\begin{aligned}
R_{3}^{(3)}(q)= & 2 i q^{-1} \mu\left(2 \tau+\frac{1}{2}, \frac{1}{2} ; 24 \tau\right)-2 q^{-\frac{1}{8}} \frac{\theta_{1,4}\left(q^{3},-q^{3}, q\right)}{\vartheta\left(\frac{1}{2} ; \tau\right)} \\
= & 2 i q^{-1} \mu\left(2 \tau+\frac{1}{2}, \frac{1}{2} ; 24 \tau\right)-\frac{2 q^{\frac{23}{8}}}{\vartheta\left(\frac{1}{2} ; \tau\right)} \frac{j^{3}\left(q^{96}, q^{288}\right)}{j\left(-1, q^{24}\right)} \\
& \times\left(\sum_{r, s=0}^{3}(-1)^{r} q^{\frac{r(r-1)}{2}+\frac{s(s+1)}{2}+5 r(s+1)+3(r+s)}\right. \\
& \left.\times \frac{j\left(q^{4(s-r)}, q^{16}\right) j\left(q^{24(r+s)+44}, q^{96}\right)}{j\left(-q^{24 r+22}, q^{96}\right) j\left(-q^{24 s+22}, q^{96}\right)}\right) \\
= & 2 i q^{-1} \mu\left(2 \tau+\frac{1}{2}, \frac{1}{2} ; 24 \tau\right)+\frac{2 i q^{\frac{223}{8}}}{\vartheta\left(\frac{1}{2} ; \tau\right)} \frac{\vartheta^{3}(96 \tau ; 288 \tau)}{\vartheta\left(\frac{1}{2} ; 24 \tau\right)} \\
& \times\left(\sum _ { r , s = 0 } ^ { 3 } \left((-1)^{r} q Q(r, s)\right.\right. \\
& \left.\left.\times \frac{\vartheta(4(s-r) \tau ; 16 \tau) \vartheta(\{24(r+s)+44\} \tau ; 96 \tau)}{\vartheta\left(\frac{1}{2}+\{24 r+22\} \tau ; 96 \tau\right) \vartheta\left(\frac{1}{2}+\{24 s+22\} \tau ; 96 \tau\right)}\right)\right) \\
:= & 2 i q^{-1} \mu\left(2 \tau+\frac{1}{2}, \frac{1}{2} ; 24 \tau\right)+T(\tau),
\end{aligned}
$$

where we defined

$$
Q(r, s):=\frac{r(r-1)}{2}+\frac{s(s+1)}{2}+5 s+6 r+5 r s,
$$

above.

Example 2.5 (The function $R_{1}^{(3)}$ ) Recall that

$$
R_{1}^{(3)}(q)=v(-q)
$$

With this information, we can show the following formula holds with $\tau \mapsto \tau+\frac{1}{2}$ (see A.2, pg. 355 of [7])

$$
R_{1}^{(3)}(q)=-2 i q^{-\frac{1}{2}} \mu(5 \tau, 3 \tau ; 12 \tau)+e^{\frac{-\pi i}{12}} q^{-\frac{1}{3}} \frac{\eta\left(\tau+\frac{1}{2}\right) \eta\left(3 \tau+\frac{1}{2}\right) \eta(12 \tau)}{\eta(2 \tau) \eta(6 \tau)}
$$




\section{Preliminary estimates for modular theta functions and Appell sums near $\tau=0$}

We collect all of the necessary estimates for the accessory objects that appear in this work near the point $\tau=0$. We have two subcategories of estimates that we need to deal with: the classical estimates that only need one error term, and the higher-order estimates that keep many error terms in the asymptotic expansion.

\subsection{Classical estimates}

Lemma 3.1 Let $\alpha \in[0,1)$, let $q:=e^{2 \pi i \tau}, q_{0}:=e^{\frac{-2 \pi i}{\tau}}$, and $k>1$ be a rational number. As $\tau \rightarrow 0$,

$$
\begin{aligned}
\vartheta(\alpha \tau ; \tau) & =\frac{-2 i \sin (\pi \alpha) q^{-\frac{\alpha^{2}}{2}} q_{0}^{\frac{1}{8}}}{\sqrt{-i \tau}}\left(1+O\left(q_{0}\right)\right), \\
\vartheta\left(\frac{1}{k}+\alpha \tau ; \tau\right) & =-\frac{q^{-\frac{\alpha^{2}}{2}} e^{\pi i \alpha\left(1-\frac{2}{k}\right)}}{\sqrt{-i \tau}} q_{0}^{\frac{1}{2 k^{2}}-\frac{1}{2 k}+\frac{1}{8}}\left(1+O\left(q_{0}^{\frac{1}{k}}\right)\right), \\
\eta(\tau) & =\frac{q_{0}^{\frac{1}{24}}}{\sqrt{-i \tau}}\left(1+O\left(q_{0}\right)\right) .
\end{aligned}
$$

Proof We begin with Eq. (3.1). Using the Jacobi product formula and Proposition 2.1.7, we have as $\tau \rightarrow 0$

$$
\begin{aligned}
\vartheta(\alpha \tau ; \tau) & =\frac{i q^{-\frac{\alpha^{2}}{2}}}{\sqrt{-i \tau}} \vartheta\left(\alpha ;-\frac{1}{\tau}\right) \\
& =\frac{e^{-\pi i \alpha} q^{-\frac{\alpha^{2}}{2}} q_{0}^{\frac{1}{8}}}{\sqrt{-i \tau}}\left(e^{2 \pi i \alpha} ; q_{0}\right)_{\infty}\left(q_{0} e^{-2 \pi i \alpha} ; q_{0}\right)_{\infty}\left(q_{0} ; q_{0}\right)_{\infty} .
\end{aligned}
$$

Thus,

$$
\begin{aligned}
\vartheta(\alpha \tau ; \tau) & =\frac{e^{-\pi i \alpha} q^{-\frac{\alpha^{2}}{2}} q_{0}^{\frac{1}{8}}}{\sqrt{-i \tau}}\left(1-e^{2 \pi i \alpha}+O\left(q_{0}\right)\right)\left(1+O\left(q_{0}\right)\right)\left(1+O\left(q_{0}\right)\right) \\
& =\frac{e^{-\pi i \alpha} q^{-\frac{\alpha^{2}}{2}} q_{0}^{\frac{1}{8}}}{\sqrt{-i \tau}}\left(1-e^{2 \pi i \alpha}+O\left(q_{0}\right)\right) \\
& =\frac{e^{-\pi i \alpha} q^{-\frac{\alpha^{2}}{2}}\left(1-e^{2 \pi i \alpha}\right) q_{0}^{\frac{1}{8}}}{\sqrt{-i \tau}}\left(1+O\left(q_{0}\right)\right) \\
& =\frac{-2 i \sin (\pi \alpha) q^{-\frac{\alpha^{2}}{2}} q_{0}^{\frac{1}{8}}}{\sqrt{-i \tau}}\left(1+O\left(q_{0}\right)\right),
\end{aligned}
$$


where the second to last steps follow from the fact that $1-e^{2 \pi i \alpha}$ is $O(1)$. Similarly for Eq. (3.2),

$$
\begin{aligned}
\vartheta\left(\frac{1}{k}+\alpha \tau ; \tau\right)= & \frac{i e^{\frac{-\pi i\left(\frac{1}{k^{2}}+\frac{2 \alpha \tau}{k}+\alpha^{2} \tau^{2}\right)}{\tau}}}{\sqrt{-i \tau}} \vartheta\left(\frac{1}{k \tau}+\alpha ; \frac{-1}{\tau}\right) \\
= & \frac{i e^{-\frac{2 \pi i \alpha}{k}} q^{-\frac{\alpha^{2}}{2}} q_{0}^{\frac{1}{2 k^{2}}}}{\sqrt{-i \tau}}\left(-i q_{0}^{\frac{1}{8}} e^{-\pi i\left(\alpha+\frac{1}{k \tau}\right)}\right) \\
& \times\left(e^{2 \pi i\left(\alpha+\frac{1}{k \tau}\right)} ; q_{0}\right)_{\infty}\left(q_{0} e^{-2 \pi i\left(\alpha+\frac{1}{k \tau}\right)} ; q_{0}\right)_{\infty}\left(q_{0} ; q_{0}\right)_{\infty} \\
\sim & \frac{q_{0}^{\frac{1}{2 k^{2}}+\frac{1}{8}+\frac{1}{2 k}} e^{-\pi i \alpha} e^{-2 \pi i \frac{\alpha}{k}}}{\sqrt{-i \tau}}\left(1-e^{2 \pi i \alpha} q_{0}^{-\frac{1}{k}}\right) \\
= & -\frac{q^{-\frac{\alpha^{2}}{2}} e^{\pi i \alpha\left(1-\frac{2}{k}\right)}}{\sqrt{-i \tau}} q_{0}^{\frac{1}{2 k^{2}}-\frac{1}{2 k}+\frac{1}{8}}\left(1+O\left(q_{0}^{\frac{1}{k}}\right)\right) .
\end{aligned}
$$

Finally, the estimate for the $\eta$-function follows directly from the transformation law in Proposition 2.1.

We also need similar estimates for the Appell function near $\tau=0$. Equation (2.1) gives

$$
\mu(5 \tau, 3 \tau ; 12 \tau)=\frac{q^{\frac{5}{2}}}{\vartheta(3 \tau ; 12 \tau)} \sum_{m \in \mathbb{Z}} \frac{(-1)^{m} q^{6 m(m+1)} q^{3 m}}{1-q^{12 m} q^{5}}
$$

Proposition 2.1.3 implies that

$$
\mu(5 \tau, 3 \tau ; 12 \tau)=-q^{2} \frac{\mu\left(\frac{5}{12}, \frac{1}{4} ;-\frac{1}{12 \tau}\right)}{\sqrt{-12 i \tau}}+\frac{h(2 \tau ; 12 \tau)}{2 i} .
$$

Before moving forward, we show that the integral $h(2 \tau ; 12 \tau)$ can be bounded by a standard Gaussian integral.

Lemma 3.2 Let $0 \leq \alpha<\frac{1}{2}$. Then as $\tau \rightarrow 0$,

$$
h(\alpha \tau ; \tau) \ll 1
$$

Proof The proof follows from the transformation law for $h$ given in Proposition 2.1.4:

$$
\begin{aligned}
h(\alpha \tau ; \tau) & =\frac{q^{\frac{\alpha^{2}}{2}}}{\sqrt{-i \tau}} h\left(\alpha ;-\frac{1}{\tau}\right)=\frac{q^{\frac{\alpha^{2}}{2}}}{\sqrt{-i \tau}} \int_{-\infty}^{\infty} \frac{e^{-\frac{\pi i w^{2}}{\tau}} e^{-2 \pi \alpha w}}{\cosh (\pi w)} \mathrm{d} w \\
& =\frac{q^{\frac{\alpha^{2}}{2}}}{\sqrt{-i \tau}}\left(\int_{0}^{\infty} \frac{e^{-\frac{\pi i w^{2}}{\tau}} e^{-2 \pi \alpha w}}{\cosh (\pi w)}-\int_{0}^{\infty} \frac{e^{-\frac{\pi i w^{2}}{\tau}} e^{2 \pi \alpha w}}{\cosh (\pi w)}\right) \mathrm{d} w
\end{aligned}
$$


which implies that

$$
|h(\alpha \tau ; \tau)| \leq\left\|\frac{q^{\frac{\alpha^{2}}{2}}}{\sqrt{-i \tau}}\right\|\left\|2 \int_{0}^{\infty} e^{-\frac{\pi i w^{2}}{\tau}}\left(\frac{e^{(2 \alpha-1) \pi w}}{1+e^{-2 \pi w}}+\frac{e^{-(2 \alpha+1) \pi w}}{1+e^{-2 \pi w}}\right) \mathrm{d} w\right\| .
$$

Since $0 \leq \alpha<\frac{1}{2}$, the term in the parentheses is bounded above by a constant. Therefore,

$$
|h(\alpha \tau ; \tau)| \ll\left\|\frac{q^{\frac{\alpha^{2}}{2}}}{\sqrt{-i \tau}}\right\|\left\|\int_{0}^{\infty} e^{-\frac{\pi i w^{2}}{\tau}} \mathrm{d} w\right\| \ll \frac{\sqrt{\tau}}{\sqrt{\tau}}=1
$$

where we used the fact that $y>0$ and that $\int_{\mathbb{R}} e^{-\frac{y w^{2}}{|\tau|^{2}}} \mathrm{~d} w=\sqrt{\frac{\pi}{y}}|\tau|$. This leads to the claimed estimate as $\tau \rightarrow 0$.

\subsection{Higher-order estimates}

The main terms in the estimates of the previous section will not be sufficient in proving the growth of the $a(n)$; thus, we need the following.

Lemma 3.3 Let $\alpha$ be as in Lemma 3.1. Then as $\tau \rightarrow 0$,

$$
\begin{aligned}
\vartheta(\alpha \tau ; \tau) & =-2 i \frac{\sin (\pi \alpha) q_{0}^{\frac{1}{8}}}{\sqrt{-i \tau}}\left(1-a_{1} q_{0}+a_{3} q_{0}^{3}+O\left(q_{0}^{4}\right)\right), \\
\vartheta\left(\frac{1}{2}+\alpha \tau ; \tau\right) & =-\frac{1}{\sqrt{-i \tau}}\left(1-2 i \cos (2 \pi \alpha) q_{0}^{\frac{1}{2}}+2 i \cos (4 \pi \alpha) q_{0}^{2}+O\left(q_{0}^{4}\right)\right),
\end{aligned}
$$

where

$$
\begin{aligned}
& a_{1}:=1+2 \cos (2 \pi \alpha), \\
& a_{3}:=1+2 \cos (2 \pi \alpha)+2 \cos (4 \pi \alpha) .
\end{aligned}
$$

Proof The proof of Eq. (3.5) follows directly by applying the technique in the proof of Lemma 3.1 and observing that

$$
\begin{aligned}
& (1-a x)\left(1-a x^{2}\right)\left(1-a x^{3}\right)\left(1-a^{-1} x\right)\left(1-a^{-1} x^{2}\right) \\
& \quad \times\left(1-a^{-1} x^{3}\right)(1-x)\left(1-x^{2}\right)\left(1-x^{3}\right) \\
& \quad=1-x-a^{-1} x-a x+a^{-1} x^{3}+x^{3}+a x^{3}+a^{2} x^{3}+a^{-2} x^{3}+O\left(x^{4}\right) .
\end{aligned}
$$


Similarly, Eq. (3.6) follows from the generic expansion

$$
\begin{aligned}
& \prod_{j=0}^{\infty}\left(1-a x^{-\frac{1}{2}} x^{j}\right) \prod_{j=0}^{\infty}\left(1-a^{-1} x^{\frac{1}{2}} x^{j}\right) \prod_{j=0}^{\infty}\left(1-x x^{j}\right) \\
& =1+a^{2}-a x^{-\frac{1}{2}}-\left(a^{3}+a^{-1}\right) x^{\frac{3}{2}}+O\left(x^{4}\right),
\end{aligned}
$$

which completes the proof.

\section{The $a(n)$}

The function $T(\tau)$, defined in Example 2.4, can be simplified greatly.

\section{Proposition 4.1}

$$
\begin{aligned}
T(\tau)= & \frac{4 i q^{40}}{\vartheta\left(\frac{1}{2} ; \tau\right)} \frac{\vartheta^{3}(96 \tau ; 288 \tau)}{\vartheta\left(\frac{1}{2} ; 24 \tau\right)} \\
& \times\left(q^{6} \frac{\vartheta(4 \tau ; 16 \tau) \vartheta(68 \tau ; 96 \tau)}{\vartheta\left(\frac{1}{2}+46 \tau ; 96 \tau\right) \vartheta\left(\frac{1}{2}+22 \tau ; 96 \tau\right)}\right. \\
& \times-q^{-47} \frac{\vartheta(12 \tau ; 16 \tau) \vartheta(20 \tau ; 96 \tau)}{\vartheta\left(\frac{1}{2}+94 \tau ; 96 \tau\right) \vartheta\left(\frac{1}{2}+22 \tau ; 96 \tau\right)} \\
& +q^{-39} \frac{\vartheta(4 \tau ; 16 \tau) \vartheta(20 \tau ; 96 \tau)}{\vartheta\left(\frac{1}{2}+46 \tau ; 96 \tau\right) \vartheta\left(\frac{1}{2}+70 \tau ; 96 \tau\right)} \\
& \left.\times-q^{-52} \frac{\vartheta(4 \tau ; 16 \tau) \vartheta(68 \tau ; 96 \tau)}{\vartheta\left(\frac{1}{2}+94 \tau ; 96 \tau\right) \vartheta\left(\frac{1}{2}+70 \tau ; 96 \tau\right)}\right) .
\end{aligned}
$$

\section{Proof Let}

$$
\begin{aligned}
\bar{S}(\tau) & :=\sum_{r, s=0}^{3}(-1)^{r} q^{Q(r, s)} \cdot \frac{\vartheta(4(s-r) \tau ; 16 \tau) \vartheta((24(r+s)+44) \tau ; 96 \tau)}{\vartheta\left(\frac{1}{2}+(24 r+22) \tau ; 96 \tau\right) \vartheta\left(\frac{1}{2}+(24 s+22) \tau ; 96 \tau\right)} \\
& :=\sum_{r, s=0}^{3} \varsigma(r, s ; \tau) .
\end{aligned}
$$

It is clear that $\varsigma(r, r ; \tau)=0$ since $\vartheta(0 ; 16 \tau)=0$. Furthermore, $Q(r, s)=Q(s, r)$. Using the fact that $\vartheta(-4(s-r) \tau ; 16 \tau)=-\vartheta(4(s-r) \tau ; 16 \tau)$, we deduce that

$$
\varsigma(s, r ; \tau)=-(-1)^{r+s} \varsigma(r, s ; \tau) .
$$

This tells us that we can write 


$$
\bar{S}(q)=2(\varsigma(1,0 ; \tau)+\varsigma(2,1 ; \tau)+\varsigma(3,0 ; \tau)+\varsigma(3,2 ; \tau)),
$$

which completes the proof.

Using this simplification in Proposition 4.1, we will investigate the asymptotic growth of the coefficients of $R_{3}^{3}$ in the next section.

\subsection{The pole at $\tau=\frac{1}{2}$}

As was claimed in Sect. 3.2, we require higher-order asymptotic expansions to accurately determine the growth of the $a(n)$. We break the study near $\tau=\frac{1}{2}$ into two parts: $T(\tau)$ and the Appell function, where Lemma 3.3 will prove useful for the study of $T(\tau)$.

\subsection{1 $T(\tau)$ near $\tau=\frac{1}{2}$}

The first result involves the function $\vartheta\left(\frac{1}{2} ; \tau\right)$, appearing in the denominator of $T(\tau)$. First recall the eta multiplier, given by (See Theorem 5.8.1 of [9])

$$
\varepsilon(M):= \begin{cases}\left(\frac{d}{|c|}\right) e^{\frac{\pi i}{12}\left((a+d-3) c-b d\left(c^{2}-1\right)\right)} & \text { if } c \text { is odd, } \\ \left(\frac{c}{|d|}\right) e^{\frac{\pi i}{12}\left((a-2 d) c-b d\left(c^{2}-1\right)+3 d-3\right)} & \text { if } c \text { is even, }\end{cases}
$$

where $M:=\left(\begin{array}{ll}a & b \\ c & d\end{array}\right)$ and $(\bullet)$ is the Jacobi symbol.

Now we can prove the following:

Lemma 4.2 Define $w:=\tau-\frac{1}{2}$. As $w \rightarrow 0$, we have

$$
\vartheta\left(\frac{1}{2} ; \tau\right)=2 \frac{e^{\frac{\pi i}{8}} e^{-\frac{\pi i}{16 w}}}{\sqrt{-2 w}}\left(1+O\left(e^{-\frac{\pi i}{2 w}}\right)\right) .
$$

Proof Let $z:=-\frac{1}{2 k w}-\frac{1}{k}$ and define the matrices

$$
A:=\left(\begin{array}{ll}
1 & 0 \\
2 & 1
\end{array}\right), \quad B:=\left(\begin{array}{ll}
1 & 0 \\
1 & 1
\end{array}\right) .
$$

Then, since we have the well-known formula: $\vartheta\left(\frac{1}{2} ; \tau\right)=2 \frac{\eta^{2}(2 \tau)}{\eta(\tau)}$,

$$
\begin{aligned}
\vartheta\left(\frac{1}{2} ; A z\right) & =2 \frac{\eta^{2}(2 A z)}{\eta(A z)} \\
& =2 \frac{\eta^{2}(B(2 z))}{\eta(A z)}
\end{aligned}
$$




$$
\begin{aligned}
& =2 \frac{\varepsilon(B)^{2}(2 z+1)^{\frac{1}{2}} \eta^{2}(2 z)}{\varepsilon(A) \eta(z)}=2 \frac{\varepsilon(B)^{2}(2 z+1)^{\frac{1}{2}} e^{\frac{8 \pi i}{24} z}\left(e^{4 \pi i z} ; e^{4 \pi i z}\right)_{\infty}^{2}}{\varepsilon(A) e^{\frac{2 \pi i z}{24}}\left(e^{2 \pi i z} ; e^{2 \pi i z}\right)_{\infty}} \\
& =2 \frac{\varepsilon(B)^{2}(k z+1)^{\frac{1}{2}} e^{\frac{\pi i}{4}} z\left(e^{4 \pi i z} ; e^{4 \pi i z}\right)_{\infty}^{2}}{\varepsilon(A)\left(e^{2 \pi i z} ; e^{2 \pi i z}\right)_{\infty}}=\vartheta\left(\frac{1}{2} ; \tau\right) \\
& =2 \frac{e^{\frac{\pi i}{8}} e^{-\frac{\pi i}{16 w}}}{\sqrt{-2 w}}\left(1+O\left(e^{-\frac{\pi i}{2 w}}\right)\right)
\end{aligned}
$$

which proves the claim.

Theorem 3 Let $Q_{0}:=e^{-\frac{2 \pi i}{\tau-\frac{1}{2}}}$ and write $\tau=x+i y$. Define $y:=\frac{1}{\sqrt{192 n}}$ and let $M>0$ such that $\left|x-\frac{1}{2}\right|<M y$. Then as $n \rightarrow \infty$

$$
T(\tau)=-\frac{\sqrt{3}}{6 \sqrt{\tau-\frac{1}{2}}} e^{\frac{\pi i}{4}} Q_{0}^{-\frac{1}{192}}\left(1+O\left(e^{-\pi \sqrt{\frac{n}{12}}}\right)\right) .
$$

Proof We focus our attention on

$$
\begin{aligned}
\bar{S}(\tau):= & q^{6} \frac{\vartheta(4 \tau ; 16 \tau) \vartheta(68 \tau ; 96 \tau)}{\vartheta\left(\frac{1}{2}+46 \tau ; 96 \tau\right) \vartheta\left(\frac{1}{2}+22 \tau ; 96 \tau\right)} \\
& -q^{-47} \frac{\vartheta(12 \tau ; 16 \tau) \vartheta(20 \tau ; 96 \tau)}{\vartheta\left(\frac{1}{2}+94 \tau ; 96 \tau\right) \vartheta\left(\frac{1}{2}+22 \tau ; 96 \tau\right)} \\
& +q^{-39} \frac{\vartheta(4 \tau ; 16 \tau) \vartheta(20 \tau ; 96 \tau)}{\vartheta\left(\frac{1}{2}+46 \tau ; 96 \tau\right) \vartheta\left(\frac{1}{2}+70 \tau ; 96 \tau\right)} \\
& -q^{-52} \frac{\vartheta(4 \tau ; 16 \tau) \vartheta(68 \tau ; 96 \tau)}{\vartheta\left(\frac{1}{2}+94 \tau ; 96 \tau\right) \vartheta\left(\frac{1}{2}+70 \tau ; 96 \tau\right)} .
\end{aligned}
$$

We refer to the $\vartheta$-quotient with $q^{6}$ out front as $S_{1}$, the one with $-q^{52}$ as $S_{4}$, the one with $-q^{-47}$ as $S_{2}$, and the one with $q^{-39}$ as $S_{3}$. That is, $\bar{S}(\tau)=S_{1}(\tau)+S_{2}(\tau)+$ $S_{3}(\tau)+S_{4}(\tau)$. Notice that

$$
\begin{aligned}
& S_{1}\left(\tau+\frac{1}{2}\right)+S_{4}\left(\tau+\frac{1}{2}\right)=S_{1}(\tau)+S_{4}(\tau), \\
& S_{2}\left(\tau+\frac{1}{2}\right)+S_{3}\left(\tau+\frac{1}{2}\right)=-S_{1}(\tau)-S_{3}(\tau) .
\end{aligned}
$$

Thus, we can capture the behavior near the cusp $\frac{1}{2}$ by investigating the behavior near 0 . We can apply Lemma 3.3 to the $S_{i}(\tau)$, and we find that as $\tau \rightarrow 0$

$$
S_{1}(\tau)+S_{4}(\tau)=-4 \sqrt{6} \sin \left(\frac{\pi}{4}\right) \sin \left(\frac{17 \pi}{24}\right) q_{0}^{\frac{7}{8 \cdot 96}}\left\{\left(a_{1}+a_{2}-c_{1}-c_{2}\right) q_{0}^{\frac{1}{192}}\right.
$$




$$
\left.-\left(1+2 \cosh \left(\frac{17 \pi}{12}\right)\right)\left(a_{1}+a_{2}-c_{1}-c_{2}\right) q_{0}^{\frac{3}{2 \cdot 96}}+O\left(q_{0}^{\frac{5}{192}}\right)\right\}
$$

where

$$
\begin{aligned}
& a_{1}:=2 i \cos \left(\frac{23 \pi}{24}\right), \quad a_{2}:=2 i \cos \left(\frac{11 \pi}{24}\right) \\
& c_{1}:=2 i \cos \left(\frac{47 \pi}{24}\right), \quad c_{2}:=2 i \cos \left(\frac{35 \pi}{24}\right) .
\end{aligned}
$$

Similarly as $\tau \rightarrow 0$,

$$
\begin{aligned}
S_{2}(\tau)+S_{3}(\tau)= & -4 \sqrt{6} \sin \left(\frac{\pi}{4}\right) \sin \left(\frac{5 \pi}{24}\right) q_{0}^{\frac{7}{8 \cdot 96}}\left\{\left(h_{1}+h_{2}-l_{1}-l_{2}\right) q_{0}^{\frac{1}{192}}\right. \\
& \left.-\left(1+2 \cosh \left(\frac{5 \pi}{12}\right)\right)\left(h_{1}+h_{2}-l_{1}-l_{2}\right) q_{0}^{\frac{3}{2 \cdot 96}}+O\left(q_{0}^{\frac{5}{192}}\right)\right\}
\end{aligned}
$$

where

$$
\begin{array}{ll}
h_{1}:=2 i \cos \left(\frac{23 \pi}{24}\right), & h_{2}:=2 i \cos \left(\frac{35 \pi}{24}\right) \\
l_{1}:=2 i \cos \left(\frac{47 \pi}{24}\right), & l_{2}:=2 i \cos \left(\frac{11 \pi}{24}\right) .
\end{array}
$$

We then observe that

$$
\begin{aligned}
& \sin \left(\frac{\pi}{4}\right) \sin \left(\frac{17 \pi}{24}\right)\left\{a_{1}+a_{2}-c_{1}-c_{2}\right\} \\
& \quad-\sin \left(\frac{\pi}{4}\right) \sin \left(\frac{5 \pi}{24}\right)\left\{h_{1}+h_{2}-l_{1}-l_{2}\right\}=0
\end{aligned}
$$

and

$$
\begin{aligned}
& \sin \left(\frac{17 \pi}{24}\right)\left(1+2 \cosh \left(\frac{17 \pi}{12}\right)\right)\left\{a_{1}+a_{2}-c_{1}-c_{2}\right\} \\
& -\sin \left(\frac{5 \pi}{24}\right)\left(1+2 \cosh \left(\frac{5 \pi}{12}\right)\right)\left\{h_{1}+h_{2}-l_{1}-l_{2}\right\} \\
& \quad=2 \sqrt{2} i .
\end{aligned}
$$


Thus,

$$
\begin{aligned}
\bar{S}(q \rightarrow-1) & =S_{1}(q \rightarrow 1)-S_{2}(q \rightarrow 1)-S_{3}(q \rightarrow 1)+S_{4}(q \rightarrow 1) \\
& =\left(4 \sqrt{6} \sin \left(\frac{\pi}{4}\right)\right)(2 \sqrt{2} i) Q_{0}^{\frac{19}{8 \cdot 96}}\left(1+O\left(Q_{0}^{\frac{1}{96}}\right)\right) \\
& =8 \sqrt{6} i Q_{0}^{\frac{19}{8 \cdot 96}}\left(1+O\left(Q_{0}^{\frac{1}{96}}\right)\right) .
\end{aligned}
$$

We now turn our attention to the outside $\vartheta$-quotient on $T(\tau)$. Sending $\tau \rightarrow \tau+\frac{1}{2}$ and using the appropriate $\vartheta$ transformations, we have

$$
\frac{4 i q^{\frac{223}{8}}}{\vartheta\left(\frac{1}{2} ; \tau\right)} \frac{\vartheta^{3}(96 \tau ; 288 \tau)}{\vartheta\left(\frac{1}{2} ; 24 \tau\right)} \rightarrow \frac{4 i q^{\frac{223}{8}} e^{\frac{223 \pi i}{8}}}{\vartheta\left(\frac{1}{2} ; \tau+\frac{1}{2}\right)} \frac{\vartheta^{3}(96 \tau ; 288 \tau)}{\vartheta\left(\frac{1}{2} ; 24 \tau\right)}
$$

Applying Lemmas 4.2 and 3.1 leads to the near $\frac{1}{2}$ estimate

$$
\begin{aligned}
& \frac{4 i q^{\frac{223}{8}} e^{\frac{223 \pi i}{8}}}{\vartheta\left(\frac{1}{2} ; \tau+\frac{1}{2}\right)} \frac{\vartheta^{3}(96 \tau ; 288 \tau)}{\vartheta\left(\frac{1}{2} ; 24 \tau\right)} \\
& \sim 4 i \frac{\left(-2 i \sin \left(\frac{\pi}{3}\right)\right)^{3} Q_{0}^{\frac{1}{8 \cdot 96}}}{\left(-288 i\left(\tau-\frac{1}{2}\right)\right)^{\frac{3}{2}}} \frac{\sqrt{-2\left(\tau-\frac{1}{2}\right)} Q_{0}^{-\frac{1}{32}} e^{\frac{111 \pi i}{4}}}{2}\left(-\sqrt{-24 i\left(\tau-\frac{1}{2}\right)}\right) \\
& =-\frac{\sqrt{2} e^{\frac{111 \pi i}{4}}}{96 \sqrt{\tau-\frac{1}{2}}} Q_{0}^{\frac{1}{8.96}-\frac{1}{32}} .
\end{aligned}
$$

Combining Eqs. (4.2) and (4.3), we obtain the full estimate for $T(\tau)$ near $\tau=\frac{1}{2}$ :

$$
\begin{aligned}
T\left(\tau \rightarrow \frac{1}{2}\right) & =-\frac{\sqrt{3} i}{6 \sqrt{\tau-\frac{1}{2}}} e^{\frac{111 \pi i}{4}} Q_{0}^{\frac{20}{8 \cdot 96}-\frac{24}{8 \cdot 96}}\left(1+O\left(Q_{0}^{\frac{1}{96}}\right)\right) \\
& =\frac{\sqrt{3}}{6 \sqrt{\tau-\frac{1}{2}}} e^{\frac{109 \pi i}{4}} Q_{0}^{-\frac{1}{192}}\left(1+O\left(Q_{0}^{\frac{1}{96}}\right)\right),
\end{aligned}
$$

which proves the claim.

\subsubsection{The Appell sum near $\tau=\frac{1}{2}$}

In order to simplify our calculations, we introduce the notation $\doteq$ to mean equal up to a constant multiple and $\dot{\sim}$ to mean asymptotic up to a constant multiple. Since our 
Appell sum is invariant under the transformation $\tau \mapsto \tau+\frac{1}{2}$, it suffices to look at the behavior near $\tau=0$. Thus, using the transformation law of Proposition 2.1.3,

$$
\mu\left(2 \tau+\frac{1}{2}, \frac{1}{2} ; 24 \tau\right)=-\frac{q^{\frac{1}{12}}}{\sqrt{-24 i \tau}} \mu\left(\frac{1}{12}+\frac{1}{48 \tau}, \frac{1}{48 \tau} ;-\frac{1}{24 \tau}\right)+\frac{h(2 \tau ; 24 \tau)}{2 i}
$$

Looking solely at the remaining Appell sum gives

$$
\begin{aligned}
& \mu\left(\frac{1}{12}+\frac{1}{48 \tau}, \frac{1}{48 \tau} ;-\frac{1}{24 \tau}\right) \\
& =\frac{e^{\frac{\pi i}{12}} e^{\frac{\pi i}{48 \tau}}}{\vartheta\left(\frac{1}{48 \tau} ;-\frac{1}{24 \tau}\right)} \sum_{n \in \mathbb{Z}} \frac{(-1)^{n} q_{0}^{\frac{n^{2}+n}{48}} q_{0}^{-\frac{n}{48}}}{1-e^{\frac{\pi i}{6}} q_{0}^{-\frac{1}{48}} q_{0}^{\frac{n}{24}}} \\
& \doteq \frac{q_{0}^{-\frac{1}{96}}}{q_{0}^{\frac{1}{8 \cdot 24}} q_{0}^{\frac{1}{96}}\left(1-q_{0}^{-\frac{1}{48}}+O\left(q_{0}^{\frac{1}{48}}\right)\right)}\left(-\frac{e^{-\frac{\pi i}{6}} q_{0}^{\frac{1}{48}}}{1-e^{-\frac{\pi i}{6}} q_{0}^{\frac{1}{48}}}+O\left(q_{0}^{\frac{1}{24}}\right)\right) \\
& \doteq \frac{q_{0}^{-\frac{1}{96}} q_{0}^{\frac{1}{48}}}{q_{0}^{\frac{1}{8 \cdot 24}} q_{0}^{-\frac{1}{96}}\left(1+O\left(q_{0}^{\frac{1}{48}}\right)\right)}\left(1+O\left(q_{0}^{\frac{1}{48}}\right)\right) \dot{\sim}_{0}^{\frac{1}{64}} .
\end{aligned}
$$

Plugging this back into Eq. (4.4) and using the estimate in Lemma 3.2, we find as $\tau \rightarrow 0$,

$$
\mu\left(2 \tau+\frac{1}{2}, \frac{1}{2} ; 24 \tau\right) \ll 1
$$

Thus, we have the following.

Remark 4.3 The growth of $R_{3}^{(3)}$ near $\tau=\frac{1}{2}$ is determined by the estimate in Theorem 3.

\subsection{Growth away from $\tau=\frac{1}{2}$}

We now want to show that the growth at the other cusps is negligible to that given in Theorem 3 . Thus, our target is to beat the bound exponentially

$$
\frac{Q_{0}^{-\frac{1}{192}}}{\sqrt{\tau-\frac{1}{2}}} \ll n^{\frac{1}{4}} e^{\pi \sqrt{\frac{n}{48}}}
$$


This is because when we do the integration in Sect. 4.4, we can bound the integral (for generic bounds $a$ )

$$
\int_{-a}^{a} e^{-2 \pi i \tau} e^{\pi \sqrt{\frac{n}{48}}-\varepsilon} \mathrm{d} x \ll e^{\pi \sqrt{\frac{n}{12}}-\varepsilon}
$$

for some $\varepsilon>0$. Thus, we can incorporate the estimates away from $\frac{1}{2}$ into an error term and ignore them in our final estimate for the $a(n)$.

\subsection{Bounding the Appell sum away from $\tau=\frac{1}{2}$}

Lemma 4.4 Let $|x|>M y$, and let $a$ and $b$ be positive integers with $a<b$. Furthermore, let $y:=\frac{1}{\delta \sqrt{n}}$ with $\delta>0$ and for $M>0$ define the term $\varepsilon:=-\frac{1}{\sqrt{1+M^{2}}}+1>0$. Then as $n \rightarrow \infty$

$$
\begin{aligned}
& \frac{1}{\vartheta(a \tau ; b \tau)} \ll \frac{1}{n^{\frac{1}{4}}} e^{\frac{3 \delta \sqrt{n}}{2 \pi b}\left(\frac{\pi^{2}}{6}-\varepsilon\right)}, \\
& \frac{1}{\vartheta\left(\frac{1}{2}+a \tau ; b \tau\right)} \ll \frac{1}{n^{\frac{1}{4}}} e^{\frac{3 \delta \sqrt{n}}{2 \pi b}\left(\frac{\pi^{2}}{6}-\varepsilon\right),}
\end{aligned}
$$

and

$$
\frac{1}{\vartheta\left(\frac{1}{2} ; b \tau\right)} \ll \frac{1}{n^{\frac{3}{4}}} e^{\frac{\delta \sqrt{n}}{\pi b}\left(\frac{\pi^{2}}{6}-\varepsilon\right)}
$$

Remark 4.5 The bounds above also hold for the functions $\vartheta(a \tau ; b \tau), \vartheta\left(\frac{1}{2} ; b \tau\right)$ and $\vartheta\left(\frac{1}{2}+a \tau ; b \tau\right)$ which can by seen by replacing $\log (\bullet)$ with $-\log (\bullet)$ in the proof below. We will use this fact later on in Sect. 4.3.1.

Proof The proof uses the same ideas as in $[6,10,12]$ to prove their bounds away from the dominant pole. We recall the Taylor expansion for $\log (1-z)=-\sum_{n \geq 1} \frac{z^{n}}{n}$. This implies

$$
\log \left(\frac{1}{\left(q^{a} ; q^{b}\right)_{\infty}\left(q^{b-a} ; q^{b}\right)_{\infty}\left(q^{b} ; q^{b}\right)_{\infty}}\right)=\sum_{n \geq 1} \frac{q^{a n}+q^{(b-a) n}+q^{b n}}{n\left(1-q^{b n}\right)}
$$

The trick now, as described by many works such as $[6,10,12]$, is to extract the first term in the sum and add an extra term, which will be the first term in the expansion for

$$
\log \left(\frac{1}{\left(|q|^{a} ;|q|^{b}\right)_{\infty}\left(|q|^{b-a} ;|q|^{b}\right)_{\infty}\left(|q|^{b} ;|q|^{b}\right)_{\infty}}\right)
$$


Explicitly, we have

$$
\begin{aligned}
& \sum_{n \geq 1} \frac{q^{a n}+q^{(b-a) n}+q^{b n}}{n\left(1-q^{b n}\right)} \\
& =\sum_{n \geq 2} \frac{q^{a n}+q^{(b-a) n}+q^{b n}}{n\left(1-q^{b n}\right)}+\frac{q^{a}+q^{b-a}+q^{b}}{\left(1-q^{b}\right)} \\
& \quad+\left(|q|^{a}+|q|^{b-a}+|q|^{b}\right)\left(\frac{1}{1-|q|^{b}}-\frac{1}{1-|q|^{b}}\right) .
\end{aligned}
$$

Taking the absolute value of this equation and using the fact that $1-|q|^{b} \leq\left|1-q^{b}\right|$, we have the upper bound

$$
\begin{aligned}
& \log \left(\frac{1}{\left(q^{a} ; q^{b}\right)_{\infty}\left(q^{b-a} ; q^{b}\right)_{\infty}\left(q^{b} ; q^{b}\right)_{\infty}}\right) \mid \\
\leq & \mid \sum_{n \geq 2} \frac{q^{a n}+q^{(b-a) n}+q^{b n}}{n\left(1-q^{b n}\right)}+\frac{q^{a}+q^{b-a}+q^{b}}{\left(1-q^{b}\right)} \\
& +\left(|q|^{a}+|q|^{b-a}+|q|^{b}\right)\left(\frac{1}{1-|q|^{b}}-\frac{1}{1-|q|^{b}}\right) \mid \\
\leq & \sum_{n \geq 2} \frac{|q|^{a n}+|q|^{(b-a) n}+|q|^{b n}}{n\left(1-|q|^{b n}\right)}+\frac{|q|^{a}+|q|^{b-a}+|q|^{b}}{\left|\left(1-q^{b}\right)\right|} \\
& +\left(|q|^{a}+|q|^{b-a}+|q|^{b}\right)\left(\frac{1}{1-|q|^{b}}-\frac{1}{1-|q|^{b}}\right) \\
= & \left.\sum_{n \geq 1} \frac{|q|^{a n}+|q|^{(b-a) n}+|q|^{b n}+\left(|q|^{a}+|q|^{b-a}+|q|^{b}\right)\left(\frac{1}{n\left(1-|q|^{b n}\right)}-\frac{1}{1-q^{b} \mid}\right)}{1-|q|^{b}}\right) \\
= & \log \left(\frac{1}{\left(|q|^{a} ;|q|^{b}\right)_{\infty}\left(|q|^{b-a} ;|q|^{b}\right)_{\infty}\left(|q|^{b} ;|q|^{b}\right)_{\infty}}\right) \\
& +\left(|q|^{a}+|q|^{b-a}+|q|^{b}\right)\left(\frac{1}{\left|1-q^{b}\right|}-\frac{1}{1-|q|^{b}}\right) .
\end{aligned}
$$

The Log term can be estimated by the asymptotic formulas derived for the $\vartheta$-functions in Lemma 3.1. Namely, as $n \rightarrow \infty$

$$
\begin{aligned}
& \log \left(\frac{1}{\left(|q|^{a} ;|q|^{b}\right)_{\infty}\left(|q|^{b-a} ;|q|^{b}\right)_{\infty}\left(|q|^{b} ;|q|^{b}\right)_{\infty}}\right) \\
& \ll \log \left(\frac{\sqrt{\frac{b}{\delta \sqrt{n}}}}{2 \sin \left(\pi \frac{a}{b}\right)}\right)+\frac{\delta \pi \sqrt{n}}{4 b} \\
& \ll C_{a, b}+\log \left(n^{-\frac{1}{4}}\right)+\frac{\delta \pi \sqrt{n}}{4 b}
\end{aligned}
$$


where $C_{a, b}$ is a constant. Now we bound the fractions. Recall that we are away from the root of unity, $q=1$ corresponding to $l=0$, by the amount $|x|>M y$. Following the procedure on page 10 of [6], we can bound $\frac{1}{\left|1-q^{b}\right|}$ by using the fact that cosine is a decreasing function near 0 . Namely, as $n \rightarrow \infty$

$$
\left|1-q^{b}\right|^{2}=1-2 \cos (2 \pi b x) e^{-2 \pi y b}+e^{-4 \pi b y} \gg 4 \pi^{2} b^{2} y^{2}\left(1+M^{2}\right) .
$$

This implies that

$$
\frac{1}{\left|1-q^{b}\right|} \ll \frac{1}{2 \pi b y \sqrt{1+M^{2}}}
$$

For the other fraction, we have as $n \rightarrow \infty$

$$
\frac{1}{1-|q|^{b}} \ll \frac{1}{2 \pi b y} .
$$

Combining Eqs. (4.9), (4.10), and (4.11), we have

$$
\begin{aligned}
& \log \left(\frac{1}{\left(q^{a} ; q^{b}\right)_{\infty}\left(q^{b-a} ; q^{b}\right)_{\infty}\left(q^{b} ; q^{b}\right)_{\infty}}\right) \\
& \ll C_{a, b}+\log \left(n^{-\frac{1}{4}}\right)+\frac{\delta \pi \sqrt{n}}{4 b}+\frac{3}{2 \pi b y \sqrt{1+M^{2}}}-\frac{3}{2 \pi b y},
\end{aligned}
$$

which proves Eq. (4.5).

Equation (4.6) follows by noticing that

$$
\begin{aligned}
& \log \left(\frac{1}{\left(q^{b} ; q^{b}\right)_{\infty}\left(-q^{b-a} ; q^{b}\right)_{\infty}\left(-q^{a} ; q^{b}\right)_{\infty}}\right)=\sum_{n \geq 1} \frac{(-1)^{n}\left(q^{a}+q^{b-a}\right)+q^{b}}{n\left(1-q^{b n}\right)} \\
& \ll \log \left(\frac{1}{\left(|q|^{a} ;|q|^{b}\right)_{\infty}\left(|q|^{b-a} ;|q|^{b}\right)_{\infty}\left(|q|^{b} ;|q|^{b}\right)_{\infty}}\right) \\
& \quad+\left(|q|^{a}+|q|^{b-a}+|q|^{b}\right)\left(\frac{1}{\left|1-q^{b}\right|}-\frac{1}{1-|q|^{b}}\right),
\end{aligned}
$$

as before.

For Eq. (4.7), we have that

$$
\vartheta\left(\frac{1}{2}, \tau\right)=-q^{\frac{1}{8}}(-1 ; q)_{\infty}\left(q^{2} ; q^{2}\right)_{\infty}=-2 q^{\frac{1}{8}}(-q ; q)_{\infty}\left(q^{2} ; q^{2}\right)_{\infty}
$$

which implies 


$$
\begin{aligned}
\left|\log \left(\frac{1}{\vartheta\left(\frac{1}{2}, \tau\right)}\right)\right| & =\left|-\log \left(-\frac{q^{-1 / 8}}{2}\right)+\sum_{n \geq 1} \frac{(-1)^{n} q^{n}}{n\left(1-q^{n}\right)}+\sum_{n \geq 1} \frac{q^{2 n}}{n\left(1-q^{2 n}\right)}\right| \\
& \leq B+\sum_{n \geq 1} \frac{2|q|^{n}}{n\left(1-|q|^{n}\right)} \ll \log \left(P^{2}(|q|),\right.
\end{aligned}
$$

where $B$ is a constant and $P(q)=\frac{q^{\frac{1}{24}}}{\eta(\tau)}$. Using Lemma 4.6 of [10], we have

$$
P^{2}\left(|q|^{b}\right) \ll \frac{1}{n^{\frac{3}{4}}} e^{\frac{\delta \sqrt{n}}{\pi b}\left(\frac{\pi^{2}}{6}+\frac{1}{\sqrt{1+M^{2}}}-1\right)} .
$$

This completes the proof of Eq. (4.7).

We look at the non-normalized Appell sum

$$
\begin{aligned}
A\left(2 \tau+\frac{1}{2}, \frac{1}{2} ; 24 \tau\right) & :=\vartheta\left(\frac{1}{2} ; 24 \tau\right) \mu\left(2 \tau+\frac{1}{2}, \frac{1}{2} ; 24 \tau\right) \\
& =-q \sum_{n \in \mathbb{Z}} \frac{(-1)^{n} q^{12\left(n^{2}+n\right)}}{1+q^{24 n+2}} .
\end{aligned}
$$

The following result shows that we can bound the above sum by a classical single variable theta function, $\Theta(\tau)$. A similar result was also mentioned by the authors of [6] but was not carried out explicitly.

$$
\begin{aligned}
& \text { Proposition 4.6 Let } \Theta(\tau):=\sum_{n \in \mathbb{Z}} q^{n^{2}} \text {. Then } \\
& \qquad\left|A\left(2 \tau+\frac{1}{2}, \frac{1}{2} ; 24 \tau\right)\right| \leq \frac{\Theta(i y)}{1-|q|^{2}} .
\end{aligned}
$$

Proof Splitting the sum in Eq. (4.12) into negative and positive index and then recombining, we find

$$
\begin{aligned}
A\left(2 \tau+\frac{1}{2}, \frac{1}{2} ; 24 \tau\right)= & \frac{q}{1+q^{2}} \\
& +q \sum_{n>0}(-1)^{n} q^{12\left(n^{2}+n\right)}\left(\frac{1}{1+q^{24 n+2}}+\frac{q^{-2}}{1+q^{24 n-2}}\right) .
\end{aligned}
$$

Since $|q|<1$, we have that $1-|q| \leq|1+q|$. Combined with the fact that $|q|^{m}$ is a decreasing function in $m$, we have that 


$$
\begin{aligned}
\left|A\left(2 \tau+\frac{1}{2}, \frac{1}{2} ; 24 \tau\right)\right| & \leq \frac{1}{1-|q|^{2}}+\sum_{n>0}|q|^{12\left(n^{2}+n\right)}\left(\frac{2}{1+|q|^{24 n-2}}\right) \\
& \leq \frac{1}{1-|q|^{2}}\left(1+2 \sum_{n>0}|q|^{n^{2}}\right)=\frac{1}{1-|q|^{2}} \Theta(i y),
\end{aligned}
$$

which proves the claim.

Remark 4.7 Recall that $\Theta(\tau)$ is a holomorphic modular form for the group $\Gamma_{0}(4)$. $\Gamma_{0}(4)$ has three inequivalent cusps represented by $0, \frac{1}{2}$, and $\infty$.

Recall that in Sect. 4.1.2, we computed the estimate near 0 and $\frac{1}{2}$, which gives

$$
\mu\left(2 \tau+\frac{1}{2}, \frac{1}{2} ; 24 \tau\right) \ll \frac{1}{\sqrt{|\tau|}} .
$$

Due to Proposition 4.6 and the corresponding remark, we can see we only need to check the growth of $\Theta(\tau)$ near $\infty$. Since $\Theta(\tau)$ is modular, its growth at $\infty$ is at most $O(1)$. Since under the transformation $\tau \mapsto \tau+\frac{1}{2}$, the Jacobi theta remains unchanged, that is $\vartheta\left(\frac{1}{2} ; 24 \tau\right) \mapsto \vartheta\left(\frac{1}{2} ; 24 \tau\right)$, we can use Lemmas 4.4 and 4.6 to obtain:

Theorem 4 Let $M>0$ such that $0<y M<\left|x-\frac{1}{2}\right|$. Then there is a $\beta>0$ such that as $n \rightarrow \infty$,

$$
\mu\left(2 \tau+\frac{1}{2}, \frac{1}{2} ; 24 \tau\right) \ll \frac{e^{\pi \sqrt{\frac{n}{48}}(1-\beta)}}{n^{\frac{3}{4}}} .
$$

\subsubsection{Bounding $T(\tau)$ away from $\tau=\frac{1}{2}$}

The estimates in Lemma 4.4 are not sufficient to bound $T(\tau)$ away from the dominant pole since they do not provide accurate information about the decay of the $\vartheta$-functions near generic cusps $\frac{p}{h}$. However, we can use Lemma 4.4 in combination with generalizations of Lemmas 4.2 and 3.1 to narrow down the problematic set of cusps to 5 specific cusps. To do so, we prove a series of three lemmas that give us the growth of all of the $\vartheta$-functions near a cusp $\frac{p}{h}$. We first note that $T(\tau)$ decays rapidly near 0 .

Lemma 4.8 As $\tau \rightarrow 0$,

$$
T(\tau) \ll q_{0}^{\frac{1}{96}}
$$

Proof This follows easily from Lemma 3.1.

To deal with the other cusps, we recall the fact that $\vartheta(z ; \tau)$ is a Jacobi form of weight and index $\frac{1}{2}$. Thus, the following properties hold $[7,11]$ : 
Remark 4.9 (See Chap. 2, [7]) Let $A=\left(\begin{array}{ll}a & b \\ c & d\end{array}\right) \in \mathrm{SL}_{2}(\mathbb{Z})$ and $\lambda, k \in \mathbb{Z}$. Then

$$
\begin{gathered}
\vartheta\left(\frac{z}{c \tau+d} ; \frac{a \tau+d}{c \tau+d}\right)=\chi(A)(c \tau+d)^{\frac{1}{2}} e^{\frac{\pi i c z^{2}}{c \tau+d}} \vartheta(z ; \tau), \\
\vartheta(z+\lambda \tau+k ; \tau)=\beta(\lambda) e^{-\pi i\left(\lambda^{2} \tau+2 \lambda z\right)} \vartheta(z ; \tau),
\end{gathered}
$$

where $\chi$ and $\beta$ are multipliers.

With this, we prove a series of three lemmas beginning with a generalization of Lemma 4.2. Throughout, we will work with the variable $\sigma:=-\frac{1}{h^{2} w}-\frac{d}{h}$ for $w \in \mathbb{H}$ and $d \in \mathbb{Z}$. Notice that

$$
\lim _{w \rightarrow 0} \sigma=-\infty
$$

Lemma 4.10 Let $(p, h)=1$, with $p, h$ both positive integers. Then as $\tau \rightarrow \frac{p}{h}$ for $h$ even

$$
\vartheta\left(\frac{1}{2} ; \tau\right) \dot{\sim}\left(-h\left(\tau-\frac{p}{h}\right)\right)^{-\frac{1}{2}} e^{-\frac{\pi i}{4 h^{2}\left(\tau-\frac{p}{h}\right)}},
$$

and if $h$ is odd,

$$
\vartheta\left(\frac{1}{2} ; \tau\right) \dot{\sim}\left(-h\left(\tau-\frac{p}{h}\right)\right)^{-\frac{1}{2}}
$$

Proof Let $A:=\left(\begin{array}{ll}p & b \\ h & d\end{array}\right)$ with $b, d$ so that $A \in S L_{2}(\mathbb{Z})$, which exist since $(p, h)=1$. Define $z:=\frac{h \sigma+d}{2}$ with $\sigma:=-\frac{1}{h^{2} w}-\frac{d}{h}$ for $w \in \mathbb{H}$. Notice that

$$
A(\sigma)=\frac{p}{h}+w(p d-h b)=\frac{p}{h}+w
$$

Thus, $\lim _{w \rightarrow 0} A(\sigma)=\frac{p}{h}$. Regardless of whether $h$ is even or odd, we have using the first equation of Remark 4.9,

$$
\vartheta\left(\frac{1}{2} ; A(\sigma)\right) \doteq(h \sigma+d)^{\frac{1}{2}} e^{\pi i \frac{h^{2} \sigma+h d}{4}} \vartheta\left(\frac{h \sigma+d}{2} ; \sigma\right) .
$$

If $h$ is even, $d$ must be odd. Therefore, the second equation of 4.9 implies that Eq. (4.13) reduces to,

$$
\vartheta\left(\frac{1}{2} ; A(\sigma)\right) \doteq \vartheta\left(\frac{1}{2} ; \sigma\right)
$$


which upon using the Jacobi triple product and taking the limit $w \rightarrow 0$ gives

$$
\vartheta\left(\frac{1}{2} ; A(\sigma)\right) \dot{\sim}\left(-\frac{1}{h w}\right)^{\frac{1}{2}} e^{-\frac{\pi i}{4 h^{2} w}}
$$

Subbing in $w=\tau-\frac{p}{h}$ proves the first claim.

The second case when $h$ is odd has two separate situations to contend with, depending on whether $d$ is odd or even. Assume first that $d$ is odd. Then $\frac{h}{2}$ and $\frac{d}{2}$ are both half integers. Therefore using Remark 4.9 as before, we have

$$
\begin{aligned}
\vartheta\left(\frac{1}{2} ; A(\sigma)\right) & \doteq(h \sigma+d)^{\frac{1}{2}} e^{\pi i \frac{h^{2} \sigma}{4}} \vartheta\left(\left(\left\lfloor\frac{h}{2}\right\rfloor+\frac{1}{2}\right) \sigma+\left\lfloor\frac{d}{2}\right\rfloor+\frac{1}{2} ; \sigma\right) \\
& \doteq(h \sigma+d)^{\frac{1}{2}} e^{-\pi i \sigma\left(\left\lfloor\frac{h}{2}\right\rfloor^{2}+\left\lfloor\frac{h}{2}\right\rfloor-\frac{h^{2}}{4}\right)} \vartheta\left(\frac{\sigma+1}{2} ; \sigma\right)
\end{aligned}
$$

where $\lfloor\bullet\rfloor$ is the floor function. Using the Jacobi product again and taking the limit as $w \rightarrow 0$, we have

$$
\vartheta\left(\frac{1}{2} ; A(\sigma)\right) \dot{\sim}(-h w)^{-\frac{1}{2}} e^{\frac{\pi i}{h^{2} w}\left(-\frac{h^{2}}{4}+\left\lfloor\frac{h}{2}\right\rfloor^{2}+\left\lfloor\frac{h}{2}\right\rfloor+\frac{1}{4}\right)}=(-2 w)^{-\frac{1}{2}} .
$$

The last step follows since $h$ odd implies $\left\lfloor\frac{h}{2}\right\rfloor=\frac{h-1}{2}$. If $d$ is even, the only thing that changes in Eq. (4.14) is that $\vartheta\left(\frac{\sigma+1}{2} ; \sigma\right)$ becomes $\vartheta\left(\frac{\sigma}{2} ; \sigma\right)$, which both yield the same estimate up to a constant factor as $w \rightarrow 0$ by examining the triple product representations.

As stated at the beginning of this section, we claimed that there are only a finite number of cusps we need to check. The following proposition gives us a rough bound for this number, but it more importantly tells us that all of the cusps that could cause a large pole have $p=1$ and $h$ even.

Proposition 4.11 The only cusps that could lead to $T(\tau)$ having larger growth than that at $\frac{1}{2}$ are cusps $\frac{p}{h}$ with $(p, h)=1$ with $h$ even and $h \leq 34$.

Proof We use the notation from the proof of Theorem 3, where we saw that we could write $T(\tau)$ as

$$
T(\tau) \doteq \frac{\vartheta^{3}(96 \tau ; 288 \tau)}{\vartheta\left(\frac{1}{2} ; \tau\right) \vartheta\left(\frac{1}{2} ; 24 \tau\right)} \bar{S}(\tau)
$$

Referring back to Remark 4.5 we can use Lemma 4.4 to bound the combination

$$
D:=\vartheta^{3}(96 \tau ; 288 \tau) \bar{S}(\tau)
$$


Recalling that each one of the four terms in $\bar{S}(\tau)$ is of the form

$$
\frac{\vartheta(\bullet ; 16 \tau) \vartheta(\bullet ; 96 \tau)}{\vartheta\left(\frac{1}{2}+\bullet ; 96 \tau\right) \vartheta\left(\frac{1}{2}+\bullet ; 96 \tau\right)},
$$

and that the bounds in Lemma 4.4 only depend on the factor in the second slot, we find that

$$
\begin{aligned}
D & \ll n^{-\frac{3}{2}} e^{\sqrt{192} \sqrt{n}\left(\frac{9}{2 \cdot 288 \pi}+\frac{3}{2 \cdot 16 \pi}+\frac{9}{2 \cdot 96 \pi}\right)\left(\frac{\pi^{2}}{6}-\varepsilon\right)} \\
& =n^{-\frac{3}{2}} e^{\sqrt{196} \sqrt{n}\left(\frac{5}{32 \pi}\right)\left(\frac{\pi^{2}}{6}-\varepsilon\right)} \\
& =n^{-\frac{3}{2}} e^{\frac{5 \sqrt{192} \pi}{192} \sqrt{n}\left(1-\frac{6}{\pi^{2}} \varepsilon\right)}=n^{-\frac{3}{2}} e^{\frac{5 \pi}{2} \sqrt{\frac{n}{48}}\left(1-\frac{6}{\pi^{2}} \varepsilon\right)},
\end{aligned}
$$

where $\varepsilon=1-\frac{1}{\sqrt{1+M^{2}}}$. If we choose $M>\pi \sqrt{\frac{31}{306-31 \pi^{2}}} \approx 85.084$, then

$$
D \ll n^{-\frac{3}{2}} e^{\sqrt{\frac{n}{48}}\left(1-\frac{1}{51}\right)} .
$$

We define

$$
G:=\frac{1}{\vartheta\left(\frac{1}{2} ; \tau\right) \vartheta\left(\frac{1}{2} ; 24 \tau\right)} .
$$

If $h$ is odd, by Lemma 4.10, the product $G D$ beats the target bound. Namely,

$$
G D \ll e^{\sqrt{\frac{n}{48}}(1-\beta)},
$$

for $\beta>0$. Thus, we only need to consider even $h$. In order to beat the bound $e^{\sqrt{\frac{n}{48}}}$, we refer to Lemma 4.10 and realize that we need to find the largest even $h$ that satisfies the inequality

$$
\frac{25}{h^{2}}>\frac{1}{51},
$$

which is $h=34$.

Proposition 4.11 gives us a first estimate of the number of cusps we need to check, as well as reducing our investigation to the cusps with $p=1$. As we will see in the next two lemmas, this helps us significantly.

We now generalize the estimate in the first equation of Lemma 3.1 for functions of the form $\vartheta\left(a_{1} \tau ; b_{1} \tau\right)$.

Lemma 4.12 Let $(p, h)=1$, with $p, h \in \mathbb{N}$, and $h$ even. Let $a_{1}, b_{1} \in \mathbb{N}$ such that $h \nmid b_{1}$. Define $\alpha:=\frac{a_{1}}{b_{1}}:=\frac{\alpha_{1}}{\beta_{1}}<1$ where the second equality is in reduced form. Let $\{x\}$ denote the fractional part of $x$. If $\beta_{1} \nmid p$, then as $\tau \rightarrow \frac{p}{h}$, 
$\vartheta\left(a_{1} \tau ; b_{1} \tau\right) \dot{\sim}\left(-h b_{1}\left(\tau-\frac{p}{h}\right)\right)^{-\frac{1}{2}} e^{\frac{\pi i}{h^{2} b_{1}\left(\tau-\frac{p}{h}\right)}\left(\left(\lfloor\alpha p\rfloor^{2}+2\lfloor\alpha p\rfloor\{\alpha p\}+\{\alpha p\}\right)-\frac{1}{4}-\alpha^{2} p^{2}\right)}$.

Remark 4.13 The cases when $h$ divides $b_{1}$ reduces to the situation in the first and second equations of Lemma 3.1. The extra condition that $\beta_{1}$ does not divide $p$ forces $\left\{\frac{a_{1} p}{b_{1}}\right\} \neq 0$.

Proof Let $A$ and $\sigma$ be as in the proof of Lemma 4.10. Define $z:=\alpha(p \sigma+b)$. Then by the first equation in Remark 4.9,

$$
\vartheta(\alpha A(\sigma) ; A \sigma)=\vartheta\left(\alpha\left(\frac{p \sigma+b}{h \sigma+d}\right) ; A \sigma\right) \doteq(c \sigma+d)^{\frac{1}{2}} e^{\frac{\pi i h z^{2}}{h \sigma+d}} \vartheta(z ; \sigma) .
$$

Dealing separately with $\vartheta(z ; \sigma)$ using the second equation of Remark 4.9, we have $\vartheta(z ; \sigma)=\vartheta(\alpha p \sigma+\alpha b ; \sigma)=e^{-\pi i\left(\lfloor\alpha p\rfloor^{2} \sigma+2\lfloor\alpha p\rfloor(\{\alpha p\} \sigma+\{\alpha b\})\right)} \vartheta(\{\alpha p\} \sigma+\{\alpha b\} ; \sigma)$.

Since $\{\alpha p\}<1$, the Jacobi triple product implies as $w \rightarrow 0$

$$
\vartheta(\{\alpha p\} \sigma+\{\alpha b\} ; \sigma) \dot{\sim} e^{\frac{\pi i \sigma}{4}-\{\alpha p\} \pi i \sigma} .
$$

Therefore, $\operatorname{as} \operatorname{Im}(w) \rightarrow 0$

$$
\vartheta(z ; \sigma) \dot{\sim} e^{-\pi i\left(\lfloor\alpha p\rfloor^{2} \sigma+2\lfloor\alpha p\rfloor\{\alpha p\} \sigma\right)} e^{\frac{\pi i \sigma}{4}-\{\alpha p\} \pi i \sigma} .
$$

Subbing (4.16) back into (4.15) and computing that as $\operatorname{Im}(w) \rightarrow 0$

$$
e^{\frac{\pi i h z^{2}}{h \sigma+d}} \dot{\sim} e^{\frac{\pi i h \alpha^{2} p^{2} \sigma^{2}}{h \sigma+d}}
$$

We have

$$
\begin{aligned}
\vartheta(\alpha A(\sigma) ; A \sigma) & \dot{\sim}(-h w)^{-\frac{1}{2}} e^{\frac{\pi i h \alpha^{2} p^{2} \sigma^{2}}{h \sigma+d}} e^{-\pi i\left(\lfloor\alpha p\rfloor^{2} \sigma+2\lfloor\alpha p\rfloor\{\alpha p\} \sigma\right)} e^{\frac{\pi i \sigma}{4}-\{\alpha p\} \pi i \sigma} \\
& \dot{\sim}(-h w)^{-\frac{1}{2}} e^{\frac{\pi i}{h^{2} w}\left(\lfloor\alpha p\rfloor^{2}+2\lfloor\alpha p\rfloor\{\alpha p\}+\{\alpha p\}\right)-\frac{\pi i}{4 h^{2} w}-\frac{\pi i \alpha^{2} p^{2}}{h^{2} w}} \\
& =(-h w)^{-\frac{1}{2}} e^{\frac{\pi i}{h^{2} w}\left(\left(\lfloor\alpha p\rfloor^{2}+2\lfloor\alpha p\rfloor\{\alpha p\}+\{\alpha p\}\right)-\frac{1}{4}-\alpha^{2} p^{2}\right)}
\end{aligned}
$$

Sending $w \mapsto b_{1} w$ and then $w \mapsto \tau-\frac{p}{h}$ proves the claim.

We finally have to address functions of the form $\vartheta\left(\frac{1}{2}+a_{1} \tau ; b_{1} \tau\right)$.

Lemma 4.14 Let $p, h, a_{1}, b_{1}$, and $\alpha$ as in Lemma 4.12. If $\lambda:=\frac{h+2 \alpha p}{2}$, then

$$
\vartheta\left(\frac{1}{2}+a_{1} \tau ; b_{1} \tau\right) \dot{\sim}\left(-h\left(\tau-\frac{p}{h}\right)\right)^{-\frac{1}{2}} e^{\frac{\pi i}{h^{2} b_{1}\left(\tau-\frac{p}{h}\right)}\left(\lfloor\lambda\rfloor^{2}+2\lfloor\lambda\rfloor\{\lambda\}+\{\lambda\}-\lambda^{2}-\frac{1}{4}\right)} .
$$


Remark 4.15 The cases when $h$ divides $b$ are addressed in the first and second equations of Lemma 3.1 .

Proof Define $z:=\frac{h+2 \alpha p}{2} \sigma+\frac{d+2 \alpha b}{2}$. Then

$$
\vartheta\left(\frac{1}{2}+\alpha A(\sigma) ; A(\sigma)\right)=\vartheta\left(\frac{z}{h \sigma+d} ; A(\sigma)\right) .
$$

Using Remark 4.9, we have

$$
\begin{aligned}
\vartheta & \left(\frac{z}{h \sigma+d} ; A(\sigma)\right) \\
= & (h \sigma+d)^{\frac{1}{2}} e^{\frac{\pi i h z^{2}}{h \sigma+d}} \vartheta\left(\frac{(h+2 \alpha p)}{2} \sigma+\frac{d+2 \alpha b}{2} ; \sigma\right) \\
= & (h \sigma+d)^{\frac{1}{2}} e^{\frac{\pi i h z^{2}}{h \sigma+d}} e^{-\pi i\left(\left\lfloor\frac{h+2 \alpha p}{2}\right\rfloor^{2} \sigma+2\left\lfloor\frac{h+2 \alpha p}{2}\right\rfloor\left(\left\{\frac{h+2 \alpha p}{2}\right\} \sigma+\left\{\frac{d+2 \alpha b}{2}\right\}\right)\right)} \\
& \times \vartheta\left(\left\{\frac{h+2 \alpha p}{2}\right\} \sigma+\left\{\frac{d+2 \alpha b}{2}\right\} ; \sigma\right) .
\end{aligned}
$$

Let $\lambda:=\frac{h+2 \alpha p}{2}$. Then, as $w \rightarrow 0$

$$
\vartheta\left(\left\{\frac{h+2 \alpha p}{2}\right\} \sigma+\left\{\frac{d+2 \alpha b}{2}\right\} ; \sigma\right) \dot{\sim} e^{\frac{\pi i \sigma}{4}-\pi i\{\lambda\} \sigma},
$$

and

$$
e^{\frac{\pi i h z^{2}}{h \sigma+d}} \dot{\sim} e^{\frac{\pi i h \lambda^{2} \sigma^{2}}{h \sigma+d}}
$$

Thus, taking the limit $w \rightarrow 0$ in Eq. (4.17) yields

$$
\vartheta\left(\frac{z}{h \sigma+d} ; A(\sigma)\right) \dot{\sim}(-h w)^{-\frac{1}{2}} e^{\frac{\pi i}{h^{2} w}\left(\lfloor\lambda\rfloor^{2}+2\lfloor\lambda\rfloor\{\lambda\}+\{\lambda\}-\lambda^{2}-\frac{1}{4}\right)} .
$$

Sending $w \rightarrow b_{1} w$ and then $w \rightarrow \tau-\frac{p}{h}$ proves the claim.

We can now prove the main result of this section.

Theorem 5 Let $M>0$. For $M y<\left|x-\frac{1}{2}\right|$, there exists a $\beta>0$ such that

$$
R_{3}^{(3)}(q) \ll e^{\pi \sqrt{\frac{n}{48}}(1-\beta)} .
$$

Proof Based on the form $T(\tau)$ given in Eq. (4.19), we need to investigate functions of the form:

$$
D:=\frac{\vartheta^{3}(96 \tau ; 288 \tau) \vartheta\left(a_{1} \tau ; 16 \tau\right) \vartheta\left(a_{2} \tau ; 96 \tau\right)}{\vartheta\left(\frac{1}{2}+a_{3} \tau ; 96 \tau\right) \vartheta\left(\frac{1}{2}+a_{4} \tau ; 96 \tau\right)},
$$


Additionally, we know we need to check cusps $\frac{p}{h}$ with $h$ even, so defining $w:=\tau-\frac{p}{h}, \gamma_{i}:=a_{i} \cdot p(\bmod h)$ and $Q_{0}:=e^{-\frac{2 \pi i}{\omega}}$ we know from Lemma 4.10 that

$$
G:=\frac{1}{\vartheta\left(\frac{1}{2} ; \tau\right) \vartheta\left(\frac{1}{2} ; 24 \tau\right)} \ll|w| Q_{0}^{-\frac{25}{24 \cdot 8 h^{2}}} .
$$

Let $A$ be the residue class $\frac{p}{3}(\bmod h)$. Then we have using Lemmas 4.12, 4.14, and 3.1

$$
\begin{aligned}
& \vartheta^{3}(96 \tau ; 288 \tau) \ll \begin{cases}|w|^{-\frac{1}{2}} Q_{0}^{\frac{3}{288 \cdot 8}} & h \mid 96, \\
|w|^{-\frac{1}{2}} Q_{0}^{\frac{3}{2 \cdot 288}\left(\left(\frac{A}{h}\right)^{2}-\frac{A}{h}\right)+\frac{3}{8 \cdot 2.888}} & h \mid 288 \text { and } h \nmid 96, \\
|w|^{-\frac{1}{2}} Q_{0}^{\frac{1}{8 \cdot 96 h^{2}}} & \text { else, }\end{cases} \\
& \vartheta\left(a_{1} \tau ; 16 \tau\right) \ll \begin{cases}|w|^{-\frac{1}{2}} Q_{0}^{\frac{1}{16 \cdot 8}} & h \mid 16, \text { and } h \mid a_{1}, \\
|w|^{-\frac{1}{2}} Q_{0}^{\frac{1}{32}\left(\left(\frac{\gamma 1}{h}\right)^{2}-\frac{\gamma 1}{h}\right)+\frac{1}{8 \cdot 16}} & h \mid 16 \text { and } h \nmid a_{1}, \\
|w|^{-\frac{1}{2}} Q_{0}^{-\frac{121}{576 \cdot 192 h^{2}}} & \text { else, }\end{cases} \\
& \vartheta\left(a_{2} \tau ; 96 \tau\right) \ll\left\{\begin{array}{ll}
|w|^{-\frac{1}{2}} Q_{0}^{\frac{1}{8.96 h}} & \text { if } h a_{2}, h \mid 96, \\
|w|^{-\frac{1}{2}} Q_{0}^{\frac{1}{192}\left(\left(\frac{\gamma 2}{h}\right)^{2}-\frac{\gamma 2}{h}\right)+\frac{1}{8 \cdot 96}} & h\left|a_{2}, h\right| 96, \\
|w|^{-\frac{1}{2}} Q_{0}^{\frac{1}{h^{2} \cdot 192 \cdot 576}} & h \mid a_{2}, h \nmid 96,
\end{array} .\right. \\
& \vartheta\left(\frac{1}{2}+a_{i} \tau ; 96 \tau\right) \gg|w|^{-\frac{1}{2}} Q_{0}^{-\frac{1}{2 \cdot 96 \cdot 2304 \cdot h^{2}}}
\end{aligned}
$$

Therefore,

$$
\begin{aligned}
& \text { Eq.(4.21) } \ll|w|^{-\frac{1}{2}} Q_{0}^{-\frac{1}{8 \cdot 96 h^{2}}} \\
& \text { Eq.(4.22) } \ll|w|^{-\frac{1}{2}} Q_{0}^{-\frac{121}{576 \cdot 192 h^{2}}} \\
& \text { Eq.(4.23) } \ll|w|^{-\frac{1}{2}} Q_{0}^{-\frac{1}{h^{2} \cdot 192 \cdot 576}} \\
& \text { Eq.(4.24) } \ll|w|^{-\frac{1}{2}} Q_{0}^{-\frac{1}{2 \cdot 96 \cdot 2304 h^{2}}}
\end{aligned}
$$

Combining this with Eq. (4.18), we find

$$
T(\tau) \ll Q_{0}^{-\frac{1}{h^{2}}\left(\frac{19555}{147456}\right)}
$$


We now want to see for what $\mathrm{h}$ the inequality $\frac{1}{h^{2}}\left(\frac{19555}{147456}\right) \leq \frac{1}{192}$, is satisfied, therefore telling us what cusps we explicitly need to see where there can be growth that beats out the growth at the pole $q=-1$. This inequality is satisfied for $h \geq \frac{\sqrt{58665}}{48}$ $\approx 5.04601225$. Therefore, the only cusps we explicitly need to check now are those $\frac{p}{4}$ with $\mathrm{p}=1,3$.

which is our target bound. This means we need to check $h=4,6$ in this case.

Now assume $h \nmid 96$. Recalling some of the objects from Lemma 4.14, notice that the equation (with $\lambda_{i}:=\frac{h+2 \alpha_{i}}{2}$ )

$$
\left\lfloor\lambda_{i}\right\rfloor^{2}+2\left\lfloor\lambda_{i}\right\rfloor\left\{\lambda_{i}\right\}+\left\{\lambda_{i}\right\}-\lambda_{i}^{2}-\frac{1}{4}=\alpha^{2}+\alpha-\frac{1}{4}>0,
$$

since for us $\alpha_{i}:=\frac{a_{i}}{96}<1$ with $a_{i}=22,46,70,94$ corresponding to the $\frac{1}{2}$ shifted $\vartheta$-functions that appear in the denominator of $T(\tau)$. Using Lemmas 4.12 and 4.14 in a similar manner to equations (4.19)-(4.21) and making note of equation (4.24) we can bound $D$ as

$$
D \ll|w|^{\frac{3}{2}}
$$

Combining this with the estimate for $G$, we find that there exists $\beta>0$ such that

$$
T(\tau) \ll|w|^{-\frac{1}{2}} Q_{0}^{-\frac{25}{24 \cdot 8 h^{2}}} \ll Q_{0}^{-\frac{1}{192}+\beta}
$$

for all $h>5$.

Thus, the completion of the proof now boils down to bounding the growth of $T(\tau)$ near $\frac{1}{4}$ and $\frac{3}{4}$. For $h=4$, this is quite easy, since all of the coefficients in the modular variable of $D$ are divisible by 4 . Furthermore, all of the $a_{i}$ in the elliptic variables in the denominator of $D$ are congruent to $2(\bmod 4)$ and the elliptic variable $\frac{1}{2}+a_{i} \tau$ reduces to $a_{i} \tau$. Therefore, using the first equation of Lemma 3.1

$$
D \ll|w|^{-\frac{3}{2}} Q_{0}^{\frac{1}{128}}
$$

Furthermore,

$$
G \ll|w| Q_{0}^{-\frac{1}{128}}
$$

Thus, $T \ll|w|^{-\frac{1}{2}}$.

\subsection{Integration and proof of Theorem 1 for $a(n)$}

We follow the approach of [6] by approximating our integral with Bessel functions. We take the standard counter-clockwise path around the origin $\gamma:=\left\{e^{-2 \pi i x}: x \in\right.$ $\left.\left(-\frac{1}{2}, \frac{1}{2}\right]\right\}$. By Cauchy's theorem, we have 


$$
a(n)=\int_{\gamma} R_{3}^{(3)}(q) q^{-n} \mathrm{~d} x=I_{1}+I_{2}
$$

where

$$
\begin{aligned}
& I_{1}:=\int_{\left|x-\frac{1}{2}\right|<M y} R_{3}^{(3)}\left(e^{2 \pi i \tau}\right) e^{-2 \pi i n \tau} \mathrm{d} x, \\
& I_{2}:=\int_{\left|x-\frac{1}{2}\right|>M y} R_{3}^{(3)}\left(e^{2 \pi i \tau}\right) e^{-2 \pi i n \tau} \mathrm{d} x .
\end{aligned}
$$

Due to the bound in Theorem $5, I_{2}$ will be our main term. Applying Theorem 3 , we have that

$$
\begin{aligned}
I_{1} & =\int_{\left|x-\frac{1}{2}\right|<M y} T(\tau) e^{-2 \pi i n \tau} \mathrm{d} x \\
& =-\frac{e^{\frac{\pi i}{4}} \sqrt{3}}{6} \int_{\left|x-\frac{1}{2}\right|<M y} \frac{Q_{0}^{-\frac{1}{192}}}{\sqrt{\tau-\frac{1}{2}}}\left(1+O\left(e^{-\pi \sqrt{\frac{n}{12}}}\right)\right) e^{-2 \pi i \tau n} \mathrm{~d} x \\
& =\frac{e^{\frac{\pi i}{4}} \sqrt{3}}{6} \int_{\left|x-\frac{1}{2}\right|<M y} \frac{e^{\frac{\pi i}{96\left(\tau-\frac{1}{2}\right)}}}{\sqrt{\tau-\frac{1}{2}}} e^{-2 \pi i \tau n} \mathrm{~d} x+E_{1},
\end{aligned}
$$

where $E_{1} \ll n^{\frac{1}{4}}$. Dealing with the remaining integral, we use the substitution $w:=$ $\tau+\frac{1}{2}$, and then $w=i v y$ (with $y=\frac{1}{\sqrt{192 n}}$ ) to obtain,

$$
\frac{e^{\frac{\pi i}{4}} \sqrt{3}}{6} \int_{\left|x-\frac{1}{2}\right|<M y} \frac{e^{\frac{\pi i}{96\left(\tau-\frac{1}{2}\right)}}}{\sqrt{\tau-\frac{1}{2}}} e^{-2 \pi i \tau n} \mathrm{~d} x=-\frac{(-1)^{n} \sqrt{3} i \sqrt{y}}{6} \mathbb{I}
$$

where we define

$$
\mathbb{I}:=\int_{1-i M}^{1+i M} \frac{e^{u\left(v+\frac{1}{v}\right)}}{\sqrt{v}} d v
$$

and $u:=\frac{\sqrt{3 n} \pi}{12}$. Lemma 7 of [6] gives the asymptotic expansion for $\mathbb{I}$ for such a $u$ :

$$
\mathbb{I}=i \frac{i \sqrt{12}}{3^{1 / 4} n^{1 / 4}} e^{\pi \sqrt{\frac{n}{12}}}+O\left(\frac{e^{\pi \sqrt{\frac{n}{12}}}}{n^{3 / 4}}\right) .
$$

Subbing back into Eq. (4.25) and sending $n \rightarrow \infty$ gives

$$
I_{1} \sim a(n) \sim(-1)^{n} \frac{\sqrt{6}}{12 \sqrt{n}} e^{\pi \sqrt{\frac{n}{12}}} .
$$




\section{The $b(n)$}

We now turn our attention to $R_{1}^{(3)}(q)=: \sum_{n \geq 0} b(n) q^{n}$. To begin, we note that the $b(n)$ form a weakly increasing sequence, which is apparent from the definition of $v(q)$.

Lemma 5.1 Let $b(n)$ denote the $n^{\text {th }}$ Fourier coefficient of the function $v(-q)$. Then the sequence $\{b(n)\}_{n=0}^{\infty}$ is weakly increasing and no $b(n)<0$.

The following Tauberian Theorem allows us to capture the growth of the $b(n)$ by only computing an estimate for the growth of $R_{1}^{(3)}$ in an angular region around $\tau=0$. The original theorem is due to Ingham [16], but we state it in a more modern form taking into account some additional technicalities regarding the growth of functions in angular regions around the origin.

Theorem 6 (see Theorem 1.1 of [8] with $\alpha=0$ ) Let $c(n)$ denote the coefficients of a power series $C(q):=\sum_{n=0}^{\infty} c(n) q^{n}$ with radius of convergence equal to 1 . Define $z:=x+i y \in \mathbb{C}$. If the $c(n)$ are non-negative, are weakly increasing, and we have as $t \rightarrow 0^{+}$that

$$
C\left(e^{-t}\right) \sim \lambda t^{\alpha} e^{\frac{A}{t}}
$$

and if for each $M>0$ such that $|y| \leq M|x|$

$$
C\left(e^{-z}\right) \ll|z|^{\alpha} e^{\frac{A}{z \mid}}
$$

with $A>0$, then as $n \rightarrow \infty$

$$
c(n) \sim \frac{\lambda A^{\frac{\alpha}{2}+\frac{1}{4}}}{2 \sqrt{\pi} n^{\frac{\alpha}{2}+\frac{3}{4}}} e^{2 \sqrt{A n}} .
$$

Remark 5.2 We will show the bound in Theorem 6 for $R_{1}^{(3)}$ as $\tau \rightarrow 0$ with $\tau \in \mathbb{H}$, which is sufficient to show the bound for general $z$ since we can define an even extension of $R_{1}^{(3)}$ into the lower half plane to get a function on all of $\mathbb{C}$. 


\subsection{Growth near $\tau=0$}

We focus on the Appell sum $\mu\left(\frac{5}{12}, \frac{1}{4} ;-\frac{1}{12 \tau}\right)$ appearing in Eq. (3.4):

$$
\begin{aligned}
& \mu\left(\frac{5}{12}, \frac{1}{4} ;-\frac{1}{12 \tau}\right) \\
& =\frac{e^{\frac{5 \pi i}{12}}}{\vartheta\left(\frac{1}{4} ;-\frac{1}{12 \tau}\right)} \sum_{n \in \mathbb{Z}} \frac{(-1)^{n} q_{0}^{\frac{n(n+1)}{24}} e^{\frac{n \pi i}{2}}}{1-e^{\frac{5 \pi i}{6}} q_{0}^{\frac{n}{12}}} \\
& =\frac{e^{\frac{5 \pi i}{12}}}{\vartheta\left(\frac{1}{4} ;-\frac{1}{12 \tau}\right)}\left\{\frac{1}{1-e^{\frac{5 \pi i}{6}}}+\sum_{n>0}\left(\frac{(-1)^{n} q_{0}^{\frac{n(n+1)}{24}} e^{\frac{\pi i n}{2}}}{1-e^{\frac{5 \pi i}{6}} q_{0}^{\frac{n}{12}}}+\frac{(-1)^{n} q_{0}^{\frac{n(n-1)}{24}} e^{-\frac{n \pi i}{2}}}{1-e^{\frac{5 \pi i}{6}} q_{0}^{\frac{-n}{12}}}\right)\right\} .
\end{aligned}
$$

The last line follows by splitting the sum into $n<0$ and $n>0$, and then swapping $n \mapsto-n$ in the sum over $n<0$. We then have,

$$
\sum_{n>0}\left(\frac{(-1)^{n} q_{0}^{\frac{n(n+1)}{24}} e^{\frac{\pi i n}{2}}}{1-e^{\frac{5 \pi i}{6}} q_{0}^{\frac{n}{12}}}+\frac{(-1)^{n} q_{0}^{\frac{n(n-1)}{24}} e^{-\frac{n \pi i}{2}}}{1-e^{\frac{5 \pi i}{6}} q_{0}^{\frac{-n}{12}}}\right)=O\left(q_{0}^{\frac{1}{12}}\right)
$$

We sub this back into Eq. (5.1) to obtain

$$
\mu\left(\frac{5}{12}, \frac{1}{4} ;-\frac{1}{12 \tau}\right)=\frac{-1}{2 i \sin \left(\frac{5 \pi}{12}\right) \vartheta\left(\frac{1}{4} ;-\frac{1}{12 \tau}\right)}\left(1+O\left(q_{0}^{\frac{1}{12}}\right)\right)
$$

We can use the triple product formula to deal with the $\vartheta$-function to find as $\tau \rightarrow 0$ that

$$
\begin{aligned}
\vartheta\left(\frac{1}{4} ;-\frac{1}{12 \tau}\right) & =-i e^{-\frac{\pi i}{4}} q_{0}^{\frac{1}{96}}\left(q_{0}^{\frac{1}{12}} ; q_{0}^{\frac{1}{12}}\right)_{\infty}\left(i ; q_{0}^{\frac{1}{12}}\right)_{\infty}\left(-i q_{0}^{\frac{1}{12}} ; q_{0}^{\frac{1}{12}}\right)_{\infty} \\
& \sim-2 \sin \left(\frac{\pi}{4}\right) q_{0}^{\frac{1}{96}} .
\end{aligned}
$$

Subbing Eqs. (5.2) and (5.3) into (3.4) and using Lemma 3.2, we have as $\tau \rightarrow 0$

$$
\begin{aligned}
\mu(5 \tau, 3 \tau ; 12 \tau) & =-q^{2} \frac{\mu\left(\frac{5}{12}, \frac{1}{4} ;-\frac{1}{12 \tau}\right)}{\sqrt{-12 i \tau}}+\frac{h(2 \tau ; 12 \tau)}{2 i} \\
& \sim-\frac{q_{0}^{-\frac{1}{96}}}{4 i \sin \left(\frac{\pi}{4}\right) \sin \left(\frac{5 \pi}{12}\right) \sqrt{-12 i \tau}} .
\end{aligned}
$$

Therefore, we can state the following. 
Theorem 7 As $\tau \rightarrow 0$, we have the estimate

$$
\mu(5 \tau, 3 \tau ; 12 \tau) \sim-\frac{e^{\frac{\pi i}{48 \tau}}}{4 i \sin \left(\frac{\pi}{4}\right) \sin \left(\frac{5 \pi}{12}\right) \sqrt{-12 i \tau}}
$$

We now show that the $\eta$-product

$$
\frac{\eta\left(\tau+\frac{1}{2}\right) \eta\left(3 \tau+\frac{1}{2}\right) \eta(12 \tau)}{\eta(2 \tau) \eta(6 \tau)}
$$

has similar growth near $\tau=0$. To get the behavior near 0 of the eta function involving the $\frac{1}{2}$ shift, we can proceed as we did in the proof of Lemma 4.2. Define the transformation

$$
A:=\left(\begin{array}{ll}
1 & 0 \\
2 & 1
\end{array}\right)
$$

with $w:=-\frac{1}{4 \tau}-\frac{1}{2}$ and then send $\tau \rightarrow 0$. We have that

$$
\eta(A \tau)=\epsilon(A)(2 \tau+1)^{\frac{1}{2}} \eta(\tau)=e^{-\frac{\pi i}{6}}(2 \tau+1)^{\frac{1}{2}} \eta(\tau) .
$$

Lemma 3.1 and Eq. (5.4) say that near 0,

$$
\begin{aligned}
& \eta(A w)=e^{-\frac{\pi i}{6}}(2 w+1)^{\frac{1}{2}} \eta(w)=e^{-\frac{\pi i}{6}}(2 w+1)^{\frac{1}{2}} e^{\frac{\pi i w}{12}}\left(e^{2 \pi i w} ; e^{2 \pi i w}\right)_{\infty} \\
& \sim e^{-\frac{5 \pi i}{24}}\left(-\frac{1}{2 \tau}\right)^{\frac{1}{2}} e^{-\frac{\pi i}{48 \tau}}=\frac{i e^{-\frac{5 \pi i}{24}}}{\sqrt{2 \tau}} q_{0}^{\frac{1}{96}} .
\end{aligned}
$$

Therefore as $\tau \rightarrow 0$ using Eq. (5.5),

$$
\begin{gathered}
\eta\left(\tau+\frac{1}{2}\right) \sim \eta(A w) \sim \frac{i e^{-\frac{5 \pi i}{24}}}{\sqrt{2 \tau}} q_{0}^{\frac{1}{96}}, \\
\eta\left(3 \tau+\frac{1}{2}\right) \sim \eta(A(3 w)) \sim \frac{i e^{-\frac{5 \pi i}{24}}}{\sqrt{6 \tau}} q_{0}^{\frac{1}{288}} .
\end{gathered}
$$

The other $\eta$-products satisfy the estimates near zero directly from Lemma $3.1 \mathrm{using}$ the substitutions $\tau \mapsto 2 \tau, \tau \mapsto 6 \tau$, and $\tau \mapsto 12 \tau$, respectively, we have as $\tau \rightarrow 0$ :

$$
\frac{\eta\left(\tau+\frac{1}{2}\right) \eta\left(3 \tau+\frac{1}{2}\right) \eta(12 \tau)}{\eta(2 \tau) \eta(6 \tau)} \sim i \frac{e^{-\frac{5 \pi i}{12}}}{\sqrt{-12 i \tau}} q_{0}^{-\frac{1}{96}} .
$$




\subsection{Proof of the estimate for the $b(n)$}

Theorem 8 Let $r(n)$ denote the coefficients of $v(-q)$. Then as $n \rightarrow \infty$,

$$
r(n) \sim\left(\frac{1}{2 \sin \left(\frac{\pi}{4}\right) \sin \left(\frac{5 \pi}{12}\right)}+1\right) \frac{e^{\pi \sqrt{\frac{n}{6}}}}{\sqrt{24 n}}
$$

Proof Combining Theorem 7 and Eq. (5.8), we have as $\tau \rightarrow 0$ that

$$
\begin{aligned}
v(-q) & =R_{1}^{3}(q) \sim 2 i \frac{q_{0}^{-\frac{1}{96}}}{4 i \sin \left(\frac{\pi}{4}\right) \sin \left(\frac{5 \pi}{12}\right) \sqrt{-12 i \tau}}+\frac{1}{\sqrt{-12 i \tau}} q_{0}^{\frac{1}{96}} \\
& =\frac{e^{\frac{\pi i}{48 \tau}}}{\sqrt{-12 i \tau}}\left(\frac{1}{2 \sin \left(\frac{\pi}{4}\right) \sin \left(\frac{5 \pi}{12}\right)}+1\right),
\end{aligned}
$$

where $q^{-\frac{1}{2}} \rightarrow 1$ in the limit $\tau \rightarrow 0$. Making the substitution $\tau:=\frac{i t}{2 \pi}$, we have that as $t \rightarrow 0^{+}$that

$$
R_{1}^{3}\left(e^{-t}\right)=\left(\frac{1}{2 \sin \left(\frac{\pi}{4}\right) \sin \left(\frac{5 \pi}{12}\right)}+1\right) \sqrt{\frac{\pi}{6}} \frac{e^{\frac{\pi^{2}}{24 t}}}{\sqrt{t}}
$$

The bound for the complex variable, $z$, in Theorem 6 is trivially satisfied by combining the estimates in Theorem 7 and Eq. (5.8).

Define $A:=\frac{\pi^{2}}{24}$ and $\lambda:=\left(\frac{1}{2 \sin \left(\frac{\pi}{4}\right) \sin \left(\frac{5 \pi}{12}\right)}+1\right) \sqrt{\frac{\pi}{6}}$. By Theorem 6 with $\alpha=\frac{1}{2}$, we have that

$$
\begin{aligned}
r(n) \sim \frac{\lambda}{2 \sqrt{\pi} n^{\frac{1}{2}}} e^{2 \sqrt{A n}} & =\left(\frac{1}{2 \sin \left(\frac{\pi}{4}\right) \sin \left(\frac{5 \pi}{12}\right)}+1\right) \sqrt{\frac{\pi}{6}} \frac{1}{2 \sqrt{\pi} \sqrt{n}} e^{\sqrt{\frac{n}{6}}} \\
& \sim\left(\frac{1}{2 \sin \left(\frac{\pi}{4}\right) \sin \left(\frac{5 \pi}{12}\right)}+1\right) \frac{e^{\pi \sqrt{\frac{n}{6}}}}{\sqrt{24 n}}
\end{aligned}
$$

which shows the claim.

\section{Conclusions and future work}

This work studied the estimates for the Fourier coefficients $a(n)$ and $b(n)$ for the base cases of the $R_{1}^{(k)}$ and $R_{3}^{(k)}$, respectively. Both of this families are mock theta families that were derived from Bailey chains in [19]. We expect that generalizing to $k>3$ 
should be doable by brute-force methods given that many of the features of the $\theta_{1, p}$ function that we encountered with $p=4$ in this work, generalize for $p>4$. Much of this can be seen in Hickerson and Mortenson's original work [15]. This includes the symmetry of the indefinite quadratic form $Q(r, s)$ that appears in the exponent of $q$ in the sum of $\theta_{1, p}$, which will allow for simpler expressions for the $\theta_{1, p}$ like we found in this work. Albeit possible to do without, it would be nice to find more elegant methods for dealing with the asymptotics for these families of Bailey mock theta functions. Based on numerical checks of the Fourier coefficients, we expect that the $R_{1}^{(k)}$ have weakly increasing coefficients for $k>3$.

Conjecture 1 The $R_{1}^{(k)}$ have weakly increasing coefficients for all $k \geq 3$.

Proving this by purely combinatorial means seems difficult, but possible using the many representations of $R_{1}^{(k)}$ given by Lovejoy and Osburn in [19]. One such way may involve appealing to some generalized $q$-binomial theorems and formulae for Gauss sums, like those posed in [17]. Such an idea seems reasonable since the $B_{k}$ in the definition of $R_{k}^{(1)}$ can be expressed as weighted sums of Gaussian polynomials. For example,

$$
\begin{aligned}
& B_{4}\left(n_{4}, n_{3}, n_{2}, n_{1} ; q\right) \\
& =(-1)^{n_{1}} q^{\frac{n_{3}\left(n_{3}+1\right)}{2}+n_{2}+2 n_{1}} \frac{(-q)_{n_{3}}(-q)_{2 n_{2}}\left(-q^{2} ; q^{2}\right)_{2 n_{1}}}{(q)_{n_{4}-n_{3}}\left(q^{2} ; q^{2}\right)_{n_{3}-n_{2}}\left(q^{4} ; q^{4}\right)_{n_{2}-n_{1}}\left(q^{8} ; q^{8}\right)_{n_{1}}} \\
& =(-1)^{n_{1}} q^{\frac{n_{3}\left(n_{3}+1\right)}{2}+n_{2}+2 n_{1}}\left[\begin{array}{l}
n_{4} \\
n_{3}
\end{array}\right]_{q}\left[\begin{array}{l}
n_{3} \\
n_{2}
\end{array}\right]_{q^{2}}\left[\begin{array}{l}
n_{2} \\
n_{1}
\end{array}\right]_{q^{4}} \frac{\left(-q ; q^{2}\right)_{n_{2}}\left(-q^{2} ; q^{4}\right)_{n_{1}}}{(q)_{n_{4}}},
\end{aligned}
$$

where $\left[\begin{array}{c}m \\ n\end{array}\right]_{q}:=\frac{(q)_{m}}{(q)_{m-n}(q)_{n}}$ is the Gaussian $q$-binomial coefficient.

Acknowledgements This work is part of an ongoing Ph.D. thesis advised by Kathrin Bringmann, and we would like to thank her for her contributions. We would like to give special thanks to Caner Nazaroglu for giving insight into many of the calculations in this work and Jeremy Lovejoy for his helpful comments and suggestions regarding many identities. We finally want to thank the anonymous reviewer, Chris JenningsSchaffer, Alexandru Ciolan, and Markus Schwagenscheidt for their helpful suggestions and edits.

Funding Open Access funding enabled and organized by Projekt DEAL.

Open Access This article is licensed under a Creative Commons Attribution 4.0 International License, which permits use, sharing, adaptation, distribution and reproduction in any medium or format, as long as you give appropriate credit to the original author(s) and the source, provide a link to the Creative Commons licence, and indicate if changes were made. The images or other third party material in this article are included in the article's Creative Commons licence, unless indicated otherwise in a credit line to the material. If material is not included in the article's Creative Commons licence and your intended use is not permitted by statutory regulation or exceeds the permitted use, you will need to obtain permission directly from the copyright holder. To view a copy of this licence, visit http://creativecommons.org/licenses/by/4.0/. 


\section{References}

1. Andrews, G.: Multiple series Rogers-Ramanujan type identities. Pac. J. Math. 114, 267-283 (1984)

2. Andrews, G.: q-Series: Their Development and Application in Analysis, Number Theory, Combinatorics, Physics, and Computer Algebra. Regional Conference Series in Mathematics, vol. 66. AMS, Providence (1986)

3. Bailey, W.N.: Some identities in combinatory analysis. Proc. Lond. Math. Soc. 49, 421-425 (1947). https://doi.org/10.1112/plms/s2-49.6.421

4. Bailey, W.N.: Identities of the Rogers-Ramanujan type. Proc. Lond. Math. Soc. 50, 1-10 (1948). https://doi.org/10.1112/plms/s2-50.1.1

5. Bringmann, K., Mahlburg, K.: Asymptotic inequalities for positive crank and rank moments. Trans. Am. Math. Soc. 366, 1073-1094 (2014). https://doi.org/10.1090/S0002-9947-2013-05945-4

6. Bringmann, K., et al.: Overpartitions with restricted odd differences. Electron. J. Combin. (2015). https://doi.org/10.37236/5248

7. Bringmann, K., et al.: Harmonic Maass Forms and Mock Modular Forms. American Mathematical Society Colloquium Publications. AMS, Providence (2017)

8. Bringmann, K., Jennings-Schaffer, C., Mahlburg, K.: On a Tauberian Theorem of Ingham and EulerMaclaurin Summation. Preprint (2020). arXiv:1910.03036

9. Cohen, H., Stroemberg, F.: Modular Forms: A Classical Approach. Graduate Studies in Mathematics. AMS, Providence (2017)

10. Dousse, J., Mertens, M.: Asymptotic formulae for partition ranks. Acta Arith. 168, 83-100 (2015). https://doi.org/10.4064/aa168-1-5

11. Eichler, M., Zagier, D.: The Theory of Jacobi Forms. Birkhäuser, Boston (1985)

12. Frnka, R.: Asymptotic formulae for mixed congruence stacks. Res. Math. Sci. 5, 8 (2018). https://doi. org/10.1007/s40687-018-0124-6

13. Gordon, M., Mcintosh, R.: A Survey of Classical Mock Theta Functions. Developmental Mathematics, pp. 95-144. Springer, New York (2011). https://doi.org/10.1007/978-1-4614-0028-8_9

14. Gu, N., Liu, J.: Families of multisums as mock theta functions. Adv. Appl. Math. 79, 98-124 (2016). https://doi.org/10.1016/j.aam.2016.04.003

15. Hickerson, D., Mortenson, E.: Hecke-type double sums, Appell-Lerch sums, and mock theta functions (I). Proc. Lond. Math. Soc. 109(2), 382-422 (2012). https://doi.org/10.1112/plms/pdu007

16. Ingham, A.E., Tauberian, A.: Theorem for partitions. Ann. Math. 42, 1075-1090 (1941). https://doi. org/10.2307/1970462

17. Kilic, E., Prodinger, H.: Evaluation of sums involving Gaussian q-binomial coefficients with rational weight functions. Int. J. Number Theory 12(2), 495-504 (2016). https://doi.org/10.1142/ S1793042116500305

18. Lovejoy, J.: Bailey pairs and indefinite quadratic forms. Math. Anal. Appl. 410, 1002-1013 (2013). https://doi.org/10.1016/j.jmaa.2013.09.009

19. Lovejoy, J., Osburn, R.: The Bailey chain and mock theta functions. Adv. Math. 238, 442-458 (2013). https://doi.org/10.1016/j.aim.2013.02.005

20. Wright, E.M.: The asymptotic expansion of the generalized Bessel function. Ann. Math. 38, 257-270 (1941). https://doi.org/10.2307/1970462

21. Zwegers, S.: Mock theta functions. PhD thesis, Utrecht (2002). arXiv:0807.4834

Publisher's Note Springer Nature remains neutral with regard to jurisdictional claims in published maps and institutional affiliations. 